TRANSACTIONS OF THE

AMERICAN MATHEMATICAL SOCIETY

Volume 352, Number 1, Pages 257-284

S 0002-9947(99)02209-6

Article electronically published on September 21, 1999

\title{
ORBIT EQUIVALENCE OF GLOBAL ATTRACTORS OF SEMILINEAR PARABOLIC DIFFERENTIAL EQUATIONS
}

\author{
BERNOLD FIEDLER AND CARLOS ROCHA
}

\begin{abstract}
We consider global attractors $\mathcal{A}_{f}$ of dissipative parabolic equations

$$
u_{t}=u_{x x}+f\left(x, u, u_{x}\right)
$$

on the unit interval $0 \leq x \leq 1$ with Neumann boundary conditions. A permutation $\pi_{f}$ is defined by the two orderings of the set of (hyperbolic) equilibrium solutions $u_{t} \equiv 0$ according to their respective values at the two boundary points $x=0$ and $x=1$. We prove that two global attractors, $\mathcal{A}_{f}$ and $\mathcal{A}_{g}$, are globally $C^{0}$ orbit equivalent, if their equilibrium permutations $\pi_{f}$ and $\pi_{g}$ coincide. In other words, some discrete information on the ordinary differential equation boundary value problem $u_{t} \equiv 0$ characterizes the attractor of the above partial differential equation, globally, up to orbit preserving homeomorphisms.
\end{abstract}

\section{INTRODUCTION AND RESULT}

On the unit interval $0 \leq x \leq 1$, the interplay of linear diffusion with nonlinear, spatially heterogeneous reaction and drift terms can be modeled by the scalar parabolic partial differential equation

$$
u_{t}=u_{x x}+f\left(x, u, u_{x}\right) .
$$

Equations of this form arise in many applied contexts. We just mention population dynamics in mathematical biology [CS80], reactor dynamics in chemical engineering [Ari75], viscosity limits to hyperbolic conservation laws [Smo83], clustering effects in astrophysics [Wol92], [Wol94], and phase transitions in materials sciences [CP89], [FH89].

The present paper aims at a global analysis of the long time behavior of the infinite dimensional dynamical system (1.1). For definiteness, we impose Neumann boundary conditions

$$
u_{x}=0, \quad \text { for } x=0,1 .
$$

Let $f$ be twice continuously differentiable: $f \in C^{2}$. By standard semigroup theory, the (local) solutions $u=u(t, x)$ of (1.1) with initial condition $u(0, x)=u_{0}(x)$ then define a dynamical system

$$
0 \leq t \mapsto u(t, \cdot) \in X
$$

Received by the editors September 17, 1996 and, in revised form, June 12, 1997. 1991 Mathematics Subject Classification. Primary 58F39, 35K55, 58F12.

(C)1999 American Mathematical Society 
see [Hen81] or [Paz83]. The state space $X$ can be picked here as the Sobolev space $H^{2}$ of $x$-profiles

$$
x \mapsto u(t, x)
$$

with Lebesgue square integrable second $x$-derivative $u_{x x}$, intersected with the appropriate Neumann conditions,

$$
X=H^{2}([0,1], \mathbb{R}) \cap\left\{u_{x}=0 \text { at } x=0,1\right\} .
$$

By Sobolev embedding, the $x$-profiles are at least once continuously differentiable, $X \subseteq C^{1}$. Moreover, the time- $t$ maps

$$
u_{0} \mapsto u\left(t, \cdot ; u_{0}\right) \in X
$$

are compact for $t>0$, by the smoothing action of diffusion.

We assume the nonlinearity $f$ to be dissipative: there exists a fixed large ball $B \subseteq X$ which attracts each solution. In particular, solutions (1.3) are defined for all $t \geq 0$, and for any $u_{0} \in X$ there exists $t_{0}=t_{0}\left(u_{0}\right) \geq 0$ such that $u(t, \cdot) \in B$, for all $t \geq t_{0}$. Then our dynamical system possesses a global attractor $\mathcal{A}_{f}$. This set can be characterized as the maximal compact invariant subset of $X$. It is also the smallest subset of $X$ which attracts all bounded sets. Equivalently, $\mathcal{A}_{f}$ consists of all those $u_{0} \in X$ which, for $t=0$, lie on an orbit $u(t, \cdot) \in X$ of (1.1) which is defined and uniformly bounded for all real times $t$, both positive and negative. See [Hal88], [Tem88], [Lad91], [BV89] for reference. We state explicit sufficient conditions for $f$ to be dissipative, which we assume to hold in this paper. There exists a constant $C_{1}>0$ such that

$$
f(x, u, 0) \cdot u<0
$$

for $|u| \geq C_{1}$, and moreover there exist continuous functions $a, b$ as well as an exponent $\gamma<2$ such that

$$
|f(x, u, p)|<a(u)+b(u)|p|^{\gamma}
$$

for all $x, u, p$. For dissipativeness under conditions (1.7), (1.8), viz. for bounds on $\sup \left|u_{x}\right|$, see [Ama85], Theorem 5.3. We defer a discussion of these rather restrictive conditions to section 8 .

Let $\mathcal{E}_{f} \subset X$ denote the set of equilibria, that is, of solutions $u \in X$ of the Neumann boundary value problem for the second order ordinary differential equation

$$
0=u_{x x}+f\left(x, u, u_{x}\right) .
$$

We assume all equilibria to be hyperbolic, that is, the linearized equation

$$
0=\eta_{x x}+f_{p} \cdot \eta_{x}+f_{u} \cdot \eta
$$

with Neumann boundary condition $\eta_{x}=0$, for $x=0,1$, possesses only the trivial solution $\eta \equiv 0$. Here the partial derivatives $f_{u}$ and $f_{p}$ of $f=f(x, u, p)$ are evaluated at $u=u(x), p=u_{x}(x)$, for equilibria $u \in \mathcal{E}_{f}$. Equivalently, the real part of all eigenvalues $\lambda$ of the linearization

$$
\lambda \eta=\eta_{x x}+f_{p} \cdot \eta_{x}+f_{n} \cdot \eta
$$

is nonzero, for all $u \in \mathcal{E}_{f}$. In fact, all eigenvalues must be real, because the SturmLiouville problem (1.11) is selfadjoint, under an appropriately weighted $L^{2}$ scalar product. We note that hyperbolicity of $\mathcal{E}_{f}$ is a generic assumption on $f$, see [Smo83], [BP87]. 
By the implicit function theorem, hyperbolic equilibria are isolated in $X$. Because $\mathcal{E}_{f} \subseteq \mathcal{A}_{f}$ is compact, the set of equilibria is finite:

$$
\mathcal{E}_{f}=\left\{u^{1}, \ldots, u^{N}\right\} .
$$

Here the numbering is chosen such that

$$
u^{1}<u^{2}<\ldots<u^{N} \text {, at } x=0 .
$$

Indeed, the equilibria are strictly ordered by their values at $x=0$, due to uniqueness of the initial value problem for (1.9) and because $u_{x}^{k}=0$ for all $k$, at $x=0$. For the same reason, the equilibria can be ordered by their other boundary value, at $x=1$. This defines a permutation $\pi=\pi_{f}$ such that

$$
u^{\pi(1)}<u^{\pi(2)}<\ldots<u^{\pi(N)}, \text { at } x=1 .
$$

Note that the permutation $\pi_{f}$ only encodes ordering information on the boundary value problem for the ordinary differential equation (1.9). We call $\pi_{f}$ the shooting permutation associated to the equilibria of $f$.

To compare the global attractors $\mathcal{A}_{f}, \mathcal{A}_{g}$ of (1.1) for different dissipative nonlinearities $f, g$, we recall the usual notion of $C^{0}$ global orbit equivalence of $\mathcal{A}_{f}$ and $\mathcal{A}_{g}$ : there exists a homeomorphism

$$
H: \quad \mathcal{A}_{f} \rightarrow \mathcal{A}_{g}
$$

which maps $f$-orbits $\{u(t, \cdot) \in X \mid t \in \mathbb{R}\} \subseteq \mathcal{A}_{f}$ onto $g$-orbits, preserving the time direction of the orbits. We use the notation

$$
\mathcal{A}_{f} \cong \mathcal{A}_{g}
$$

for global orbit equivalence. With this definition we can now state our main result.

Theorem 1.1. Let $f, g \in C^{2}$ satisfy dissipation conditions (1.7), (1.8). Assume they possess only hyperbolic equilibria with associated shooting permutations $\pi_{f}, \pi_{g}$. Then the global attractors $\mathcal{A}_{f}$ and $\mathcal{A}_{g}$ are globally orbit equivalent, $\mathcal{A}_{f} \cong \mathcal{A}_{g}$, if

$$
\pi_{f}=\pi_{g} .
$$

An outline of the proof will be given in section 2. The proof itself fills sections 3 to 7 . We conclude with a detailed discussion, in section 8 . We conclude this introduction indicating the two main features which make our theorem work: Morse structure and nodal properties. For a survey see [Fie89].

The Morse, gradient, or variational structure of (1.1) is given explicitly, for $f=f(x, u)$ independent of $p=u_{x}$, in terms of the Lyapunov functional $V$ on $X$ defined by

$$
V(u):=\int_{0}^{1}\left(\frac{1}{2}\left(u_{x}\right)^{2}-F(x, u)\right) d x .
$$

Here $F$ is a primitive function of $f$ with respect to $u$, that is, $F_{u}=f$. Inserting solutions $u(t, x)$ of $(1.1)$, we obtain

$$
\frac{d}{d t} V(u(t, \cdot))=-\int_{0}^{1}\left(u_{t}\right)^{2} d x .
$$

In particular, $V$ is bounded on the compact attractor $\mathcal{A}_{f}$, and is strictly decreasing with time, except of course on the equilibrium set $\mathcal{E}_{f}$. A similar, though less explicit, Lyapunov functional $V: \mathcal{A}_{f} \rightarrow \mathbb{R}$ with these properties also exists in the general case where $f=f(x, u, p)$ is allowed to depend on $p=u_{x}$, see [Zel68], [Mat88]. 
By its Morse structure, our global attractor $\mathcal{A}_{f}$ decomposes into equilibria $\mathcal{E}_{f}$ and their heteroclinic or connecting orbits. These orbits, by definition, limit onto (different) equilibria for $t \rightarrow+\infty$ and $t \rightarrow-\infty$, respectively. Indeed, $\frac{d}{d t} V=0$ on $\alpha$ and $\omega$-limit sets, and therefore these sets are given by a single, isolated hyperbolic equilibrium, each. In short

$$
\mathcal{A}_{f}=\mathcal{E}_{f} \cup\{\text { heteroclinic orbits }\} .
$$

Investigation of the geometry of the heteroclinic set is an ongoing topic of research. We mention [Hen81], [Hen85], [Ang86], [BF88], [BF89], [AF88], [FR91], for partial results on equations of the form (1.1). Recently, [FR96] have settled the question for which $u^{i}, u^{j} \in \mathcal{E}_{f}$ does there exist a heteroclinic connection from $u^{i}$ to $u^{j}$. The complete answer can be given by an explicit and constructive process, which only uses information on the equilibrium permutation $\pi_{f}$. The precise global geometry of the connections, however, remains unresolved.

The crucial importance of nodal properties for the qualitative understanding of equations of the form (1.1) was first noted by [Mat82]. Take any two solutions $u^{1}(t, \cdot), u^{2}(t, \cdot)$. Let $z\left(u^{1}-u^{2}\right)$, for fixed $t$, denote the number of strict sign changes (nodes) of the $x$-profile $x \mapsto u^{1}(t, x)-u^{2}(t, x)$. Then

$$
t \mapsto z\left(u^{1}(t, \cdot)-u^{2}(t, \cdot)\right)
$$

is nonincreasing with time $t$. In fact, $z\left(u^{1}(t, \cdot)-u^{2}(t, \cdot)\right)$ drops strictly whenever the $x$-profile $u^{1}-u^{2}$ possesses a multiple zero $u^{1}-u^{2}=0, u_{x}^{1}-u_{x}^{2}=0$. Indeed, the nodal property follows because the difference $\tilde{u}:=u^{1}-u^{2}$ satisfies a (time-dependent) linear parabolic equation. For comprehensive nodal properties in the linear case see the recent account by [Ang88]. The autonomous linear case is classical [Stu36], [Pol33].

Nodal properties have many important, and sometimes surprising consequences. For example, just by hyperbolicity of the equilibria $\mathcal{E}_{f}$ alone and without further genericity or nondegeneracy assumptions on $f$, our dynamical system (1.1) turns out to be Morse-Smale, see [Hen85], [Ang86]. In fact, unstable and stable manifolds $W^{u}$ and $W^{s}$ of any two equilibria necessarily intersect transversely, by the above nodal property. Together with the Morse structure this makes our dynamical MorseSmale system structurally stable: small dissipative perturbations of the nonlinearity $f$ to $\tilde{f}$ yield global attractors $\mathcal{A}_{f}, \mathcal{A}_{\tilde{f}}$ which are globally $\left(C^{0}\right)$ orbit equivalent. In finite dimensions this result is due to Palis and Smale, see [Pal69], [PS70], [PdM82]. The infinite dimensional case is due to [Oli83]; see also [HMO84].

In view of Morse-Smale stability our strategy for a proof of our theorem seems simple. If we find a homotopy $f^{\tau}, 0 \leq \tau \leq 1$, from $f^{0}=f$ to $f^{1}=g$, such that the equilibrium set $\mathcal{E}_{f^{\tau}}$ remains hyperbolic throughout, then $\mathcal{A}_{f}$ and $\mathcal{A}_{g}$ are orbit equivalent by Oliva, Palis, and Smale. Indeed we can cover the compact parameter interval $0 \leq \tau \leq 1$ by finitely many open intervals of structural stability. In the next section we explain why matters are not all that simple, in fact, and how our proof takes a few more meanders before completion.

\section{ACKNOWLEDGEMENT}

The first author gratefully acknowledges extensive all-round hospitality experienced at Instituto Superior Técnico, Lisboa. Both authors have benefitted from helpful comments by Alain Chenciner, Matthias Wolfrum, Genevieve Raugel, and Jorge Sotomayor. 
This work was supported by the Deutsche Forschungsgemeinschaft, "Globale unendlich-dimensionale Dynamik", Junta Nacional de Investigação Científica e Tecnológica (STRDA/C/CEN/528/92), and by the European Community Human Capital Programme "Nonlinear Boundary Value Problems".

\section{Outline OF PROOF}

Throughout, let the assumptions of Theorem 1.1 hold. In this section we explain our overall strategy of proof for our theorem on global orbit equivalence. Structural stability of Morse-Smale systems will be the main actor, though mostly somewhat behind the scene. We first explain the difficulty which a naive homotopy $f^{\tau}$ is facing, in the spirit of the end of the introduction section 1 . We then indicate how discretization will help, in section 3. Section 4 is devoted to normalizations which involve dissipativeness. Our crucial trick, gaining additional freedom for homotopies, is the insertion of additional, artificial discretization points, in section 5 , which essentially produce a high-dimensionally unstable suspension of the original global attractor $\mathcal{A}_{f}$ in an augmented discrete system. Sections 6 and 7 will highlight the additional freedom gained by augmented discretization. In fact, the additional freedom reduces the question of finding a suitable homotopy $f^{\tau}$, on the augmented discrete level, to a purely topological result on the fundamental group of a certain space of diffeomorphisms. With those details at hand, the outline of proof given below will then have solidified into a complete proof.

Let us first try the most naive, standard homotopy

$$
f^{\tau}=(1-\tau) f+\tau g, \quad 0 \leq \tau \leq 1,
$$

between dissipative nonlinearities $f, g$ with global attractors $\mathcal{A}_{f}, \mathcal{A}_{g}$. By dissipation condition (1.7), (1.8), global attractors respect bounds $|u| \leq C_{1},\left|u_{x}\right| \leq C_{2}$, uniformly in $t, x$. Modifying $f, g$ to become

$$
f=g=-u,
$$

for $|u| \geq 2 C_{1}$ or $\left|u_{x}\right| \geq 2 C_{2}$, as explained in section 4 below, ensures dissipativeness throughout the homotopy. The homotopy parameter $\tau$ induces a bifurcation diagram for the sets $\mathcal{E}_{f^{\tau}}$ of equilibria. If all equilibria remain hyperbolic, for all $0 \leq \tau \leq 1$, then the Morse-Smale property implies equivalence of $\mathcal{A}_{f}$ and $\mathcal{A}_{g}$. However, bifurcations of saddle-node type could occur, for some $0<\tau<1$. Saddlenodes are lethal for our Morse-Smale proof. To understand the difficulty in avoiding saddle-nodes, we use a shooting, or "time" map approach to the equilibrium ODE (1.9), alias

$$
\begin{aligned}
& u_{x}=p \\
& p_{x}=-f(x, u, p)
\end{aligned}
$$

with Neumann boundary conditions $p=0$, at $x=0,1$. At $x=0$, consider the line of initial conditions

$$
u=a, \quad p=0,
$$

parametrized by $a \in \mathbb{R}$. Letting $x$ evolve forward, according to (2.3), we obtain solutions $u(x ; a), p(x ; a)$ and the shooting curves

$$
a \mapsto S_{f}^{x}(a):=(u(x ; a), p(x ; a)) \in \mathbb{R}^{2}
$$




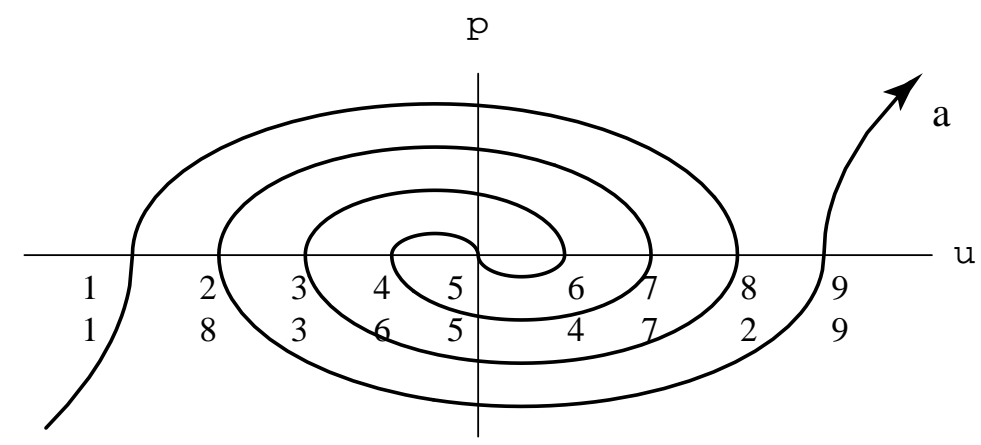

Figure 2.1. A stylized shooting curve $S_{f}^{x=1}$ for the Chafee-Infante problem $(3 \pi)^{2}<\lambda<(4 \pi)^{2}$.

in $(u, p)$ space. See for example [FR91], [Roc91], [FR96] and the references there. Each shooting curve is a planar $C^{2}$ Jordan curve. Their collection for $0 \leq x \leq 1$ defines the shooting surface

$$
S_{f}: \quad(x, a) \mapsto\left(x, S_{f}^{x}(a)\right) \in[0,1] \times \mathbb{R}^{2}
$$

in $(x, u, p)$ space. Note that the equilibria $\mathcal{E}_{f}$ are described by the values $a$ at intersections of $S_{f}^{x=1}$ with the $u$-axis:

$$
S_{f}^{x=1}(a)=(u(x=1 ; a), 0),
$$

corresponding to the Neumann boundary condition

$$
p(x=1 ; a)=0 \text {. }
$$

It turns out that $\mathcal{E}_{f}$ is hyperbolic if, and only if, $S_{f}^{x=1}$ intersects the $u$-axis transversely. If $S_{f^{\tau}}^{x=1}$ touches, and crosses the $u$-axis during a homotopy $f^{\tau}$, then a saddle-node bifurcation of equilibria occurs. Clearly, saddle-node bifurcations might occur during a naive standard homotopy $f^{\tau}=(1-\tau) f+\tau g$, with destructive consequences for the geometry of the global attractors $\mathcal{A}_{f^{\tau}}$.

There is a very special case though, where the naive, standard homotopy (2.1) works well. If the shooting surfaces of $f$ and $g$ coincide entirely,

$$
S_{f}=S_{g}
$$

then $S_{f \tau}$ is independent of $\tau$, also at $x=1$. Consequently, the global attractors $\mathcal{A}_{f}$ and $\mathcal{A}_{g}$ are then orbit equivalent. Therefore it will be sufficient to focus on just the shooting surfaces, when constructing a less naive homotopy $f^{\tau}$.

As a simple specific example, we consider the Chafee-Infante problem [Cha74], [CI74]

$$
f(x, u, p)=\lambda u\left(1-u^{2}\right)
$$

As in all dissipative systems, there exist a lowest and a highest equilibrium $\underline{u}, \bar{u}$; here $\underline{u} \equiv-1, \bar{u} \equiv+1$. In the $(u, p)$-plane the shooting curve points south-west, to the left of the lowest, and north-east, to the right of the highest equilibrium. See Figure 2.1. Observe the transverse intersections of $S_{f}^{x=1}$ with the $u$-axis corresponding to the equilibria $u^{1}, \ldots, u^{9}$. Numbering the equilibria along the shooting curve $S_{f}^{x=1}$ provides the Chafee-Infante permutation

$$
\pi_{f}=\left(\begin{array}{ll}
2 & 8
\end{array}\right)\left(\begin{array}{ll}
4 & 6
\end{array}\right)
$$


in the case of Figure 2.1. Parenthetically we note that the Morse indices, or unstable dimensions, of all equilibria are also determined by $\pi_{f}$; see again [Roc91], [FR96].

Consider a less naive homotopy $f^{\tau}$ from $f$ to $g$ next; this homotopy should preserve the transverse intersections of $S_{f^{\tau}}^{x=1}$ with the $u$-axis, enacting a MorseSmale application. Since $\pi_{f}=\pi_{g}$, by assumption, there exists a diffeotopy

$$
\varphi^{\tau}
$$

of the $(u, p)$ plane, $\varphi^{\circ}=i d$, which provides a deformation from $S_{f}^{x=1}$ to $S_{g}^{x=1}$, such that transversality of the curves to the $u$-axis is preserved throughout the homotopy. Our proof would be complete, if we could now find a homotopy $f^{\tau}$ such that for $x=1$

$$
S_{f^{\tau}}^{x}=\varphi^{\tau}\left(S_{f}^{x}\right) .
$$

But we cannot. The obstruction lies in the second order system (2.3) which constrains the evolution of shooting curves $S_{f}^{x}$. Indeed, let $\mathbf{n} \in \mathbb{R}^{2}$ denote a unit normal to $S_{f}^{x}$ at $(u, p)$, and $\sigma$ the normal speed of propagation of the curve $S_{f}^{x}$ in the direction of $\mathbf{n}$, as $x$ increases. Then $\sigma$ is the normal projection of the vector field (2.3), that is,

$$
\sigma=\mathbf{n} \cdot\left(\begin{array}{c}
p \\
-f(x, u, p)
\end{array}\right) .
$$

Varying $f$ freely, this does not impose any restriction on the speed $\sigma$, unless $\mathbf{n}=$ $(1,0)$ is horizontal. In that latter case, $S_{f}^{x}$ possesses a vertical tangent (pictorically: a nose), and

$$
\sigma=p
$$

Summarizing: higher noses move faster to the right, as $x$ increases. It is this constraint on the shape of the shooting surfaces $S_{f}, S_{g}$ which we could not overcome in our attempts to construct a homotopy $f^{\tau}$ which preserves the Morse-Smale type hyperbolicity condition (2.13) throughout $0 \leq \tau \leq 1$. Such a homotopy might in fact exist. But we did not secure a general algorithm for constructing one.

Therefore we discretize, in section 3 . The discrete equations take the form

$$
\dot{u}_{i}=f_{i}\left(u_{i-1}, u_{i}, u_{i+1}\right),
$$

$i=0, \ldots, n$, where each $f_{i}$ has strictly positive partial derivatives with respect to its off-diagonal entries $u_{i-1}$ and $u_{i+1}$. Following [FO88] we call (2.16) a Jacobi system. The Neumann boundary conditions become

$$
u_{-1}:=u_{0}, \quad u_{n+1}:=u_{n} .
$$

The global attractor of (2.16) is orbit equivalent to $\mathcal{A}_{f}$, for equidistant symmetric finite difference approximations $f_{i}$ and large enough $n$ (see section 3). Dissipativeness is also inherited (see section 4). We denote the global attractor of (2.16), (2.17) by $\mathcal{A}_{f}$, again.

We now describe the crucial trick, an $n(m-1) / 2$-dimensionally unstable suspension of $\mathcal{A}_{f}$ in a singular perturbation manner, which will gain us enough additional freedom to realize the shooting curve diffeotopy $\varphi^{\tau}$. For $i=0, \ldots, n, k=$ $1, \ldots, m-1$ and for $0<j:=i+k / m<n$ we define the augmented discrete system as

$$
\begin{array}{rlc}
\dot{u}_{i} & =f_{i}\left(u_{i-1 / m}, u_{i}, u_{i+1 / m}\right), \\
\varepsilon \dot{u}_{j} & = & u_{j-1 / m}+u_{j+1 / m} .
\end{array}
$$


Here $m \geq 5$ is chosen congruent to $1 \bmod 4$. We keep Neumann boundary conditions (2.17) in the adapted form

$$
u_{-1 / m}:=u_{0}, \quad u_{n+1 / m}:=u_{n},
$$

and let $u=\left(u_{0}, u_{1 / m}, \ldots, u_{n-1 / m}, u_{n}\right) \in \mathbb{R}^{n m+1}$. The linear $(n+1)$-dimensional subspace

$$
M:=\left\{u_{j-1 / m}+u_{j+1 / m}=0, \text { for all noninteger } 0<j<n\right\}
$$

contains all the equilibria of the flow (2.18) and is entirely composed of equilibrium points of the formally reduced system for $\varepsilon=0$ in the fast time variable $\tau=t / \varepsilon$. Note that, for the reduced flow, $M$ contains the original global attractor $\mathcal{A}_{f}$ since $m \equiv 1(\bmod 4)$ implies

$$
u_{i \pm 1 / m}=u_{i \pm 1}
$$

on $M$. For small $\varepsilon>0$, the invariant manifold $M$ is normally hyperbolic with $n(m-1) / 2$-dimensional strong unstable and strong stable fibers. By singular perturbation theory, (2.17), (2.18) possess a maximal compact invariant set $\tilde{\mathcal{A}}_{f}$ orbit equivalent to $\mathcal{A}_{f}$. These facts will be proved in section 5; see Lemma 5.1.

In section 6 we study the analogue of the shooting curves $S_{f}^{x}$ for the augmented discrete system (2.18), rewritten as a Jacobi system

$$
\dot{u}_{j}=f_{j}\left(u_{j-1 / m}, u_{j}, u_{j+1 / m}\right),
$$

$j=0,1 / m, \ldots, n$. For equilibria, $\dot{u}=0$, we obtain

$$
0=f_{j}\left(u_{j-1 / m}, u_{j}, u_{j+1 / m}\right) .
$$

Because the off-diagonal derivatives are strictly positive, these equations can be solved for $u_{j+1 / m}$, defining the shooting recursion

$$
u_{j+1 / m}=\varphi_{j}^{f}\left(u_{j-1 / m}, u_{j}\right) .
$$

Note that the partial derivative of $\varphi_{j}^{f}$ with respect to $u_{j-1 / m}$ is strictly negative. Given such a $\varphi_{j}$, we may also conversely define $f_{j}$ by $f_{j}:=u_{j+1 / m}-\varphi_{j}$, and obtain a system (2.22) with Jacobi structure.

Rewriting (2.24) as a system

$$
\begin{aligned}
\psi_{j}^{f}: \quad u_{j} & =v_{j-1 / m}, \\
v_{j} & =\varphi_{j}^{f}\left(u_{j-1 / m}, v_{j-1 / m}\right)
\end{aligned}
$$

we see that the diffeomorphisms $\psi_{j}^{f}$ replace $f$-evolution maps from $x=(j-1) / n$ to $x=j / n$, in the discretized case. Also, for non-integer $j$, the map $\psi_{j}^{f}$ is a pure rotation $R$ by 90 degrees,

$$
\psi_{j}^{f}\left(u_{j-1 / m}, v_{j-1 / m}\right)=R\left(u_{j-1 / m}, v_{j-1 / m}\right):=\left(v_{j-1 / m},-u_{j-1 / m}\right) .
$$

In particular

$$
\varphi_{j}^{f}\left(u_{j-1 / m}, v_{j-1 / m}\right)=-u_{j-1 / m}
$$

for non-integer $j$. If $j=i$ is integer, then $\psi_{i}^{f}$ was already defined by the original discretization $f_{i}$. Let the shooting quadruple

$$
\tilde{\psi}_{j}^{f}:=\psi_{j+3 / m}^{f} \circ \ldots \circ \psi_{j}^{f}
$$


be the composition of any four consecutive $\psi$-maps with non-integer $j, \ldots, j+3 / m$. Clearly $\tilde{\psi}_{j}^{f}=i d$ in this case of four rotations by 90 degrees. In Lemma 6.1 we prove that, by small perturbations of $\varphi_{j+3 / m}^{f}, \varphi_{j+2 / m}^{f}$, the shooting quadruple $\tilde{\psi}_{j}^{f}$ can be forced to realize any diffeomorphism near identity. This is the crucial additional freedom which will enable us to complete a non-naive structurally stable homotopy from $\mathcal{A}_{f}$ to $\mathcal{A}_{g}$. Indeed, between any two maps $\psi_{i}$ with integer $i$ we have now inserted $(m-1) / 4 \in \mathbb{N}$ shooting quadruples $\tilde{\psi}_{j}$, each of which can realize any near identity diffeomorphism.

Our non-naive homotopy is constructed, in section 7, as follows. Consider the diffeomorphisms

$$
\Psi_{i}^{f}:=\psi_{i}^{f} \circ \ldots \circ \psi_{0}^{f},
$$

$i=1, \ldots, n$, which accumulate diffeomorphisms $\psi_{i}^{f}, i$ integer, defined by $(2.25)$. For $g$, we define $\Psi_{i}^{g}$ analogously. Comparing with (2.13) we observe that $\Psi_{n}^{f}$ induces the permutation $\pi_{f}$, by the discrete shooting curve intersecting the diagonal transversely in the original attractor region $\{|u| \leq,|v| \leq 1\}$; see section 6 . Here we assume $n$ is chosen large enough, of course. Similarly, $\Psi_{n}^{g}$ induces $\pi_{g}=\pi_{f}$, for large $n$. In a neighborhood $\Omega$ of the attractor region, we now prescribe homotopy paths

$$
\Psi_{i}^{\tau}, \quad 0 \leq \tau \leq 1,
$$

from $\Psi_{i}^{f}=\Psi_{i}^{0}$ to $\Psi_{i}^{g}=\Psi_{i}^{1}$ in the space $\operatorname{Diff}(\Omega)$ of diffeomorphism of $\Omega$. We will assume $\Psi_{i}^{f} \equiv \Psi_{i}^{g} \equiv \Psi_{i}^{\tau}$, outside $\Omega$, without loss of generality. At the end point $i=n$, corresponding to $x=1$, we specifically prescribe $\Psi_{n}^{\tau}$ to provide a diffeotopy $\varphi^{\tau}$ of the discrete shooting curves from $f$ to $g$, analogously to the construction of $\varphi_{x=1}^{\tau}$ in the continuous case; see (2.13), (7.9). Moreover we choose a standard homotopy

$$
\psi_{i}^{\tau}:=(1-\tau) \psi_{i}^{f}+\tau \psi_{i}^{g}
$$

of diffeomorphisms (2.25) at integer values $j=i=0, \ldots, n-1$. The maps $\psi_{j}$ at noninteger values of $j$ between $n-1$ and $n$ will compensate for this mortal sin against hyperbolicity and the Morse-Smale property during the homotopy $0 \leq \tau \leq 1$.

In section 7 , Lemma 7.2 , we will prove that there exists a continuous twoparameter family $\Psi(\tau, \xi) \in \operatorname{Diff}(\Omega), 0 \leq \tau, \xi \leq 1$, of interpolating diffeomorphisms, which compensates for the mortal sin (2.31). More precisely, as indicated in Figure 2.2

$$
\begin{aligned}
& \Psi(\tau, \xi=0)=\Psi_{n-1}^{\tau}, \\
& \Psi(\tau, \xi=1)=\left(\psi_{n}^{\tau}\right)^{-1} \Psi_{n}^{\tau}, \\
& \Psi(\tau=0, \xi)=\Psi_{n-1}^{f}, \\
& \Psi(\tau=1, \xi)=\Psi_{n-1}^{g} .
\end{aligned}
$$

This fact uses essentially triviality of the fundamental group $\pi_{1}$ of the space $\operatorname{Diff}_{*}(\Omega)$ of diffeomorphisms of $\Omega$ which fix $\partial \Omega$ :

$$
\pi_{1}\left(\operatorname{Diff}_{*}(\Omega)\right)=0,
$$

by Smale's theorem. See Lemma 7.1 below.

In the final step of our proof we fix $m$ large enough to sufficiently discretize the $\xi$-variable in the interpolating family of diffeomorphisms $\Psi(\tau, \xi)$. In fact, all 


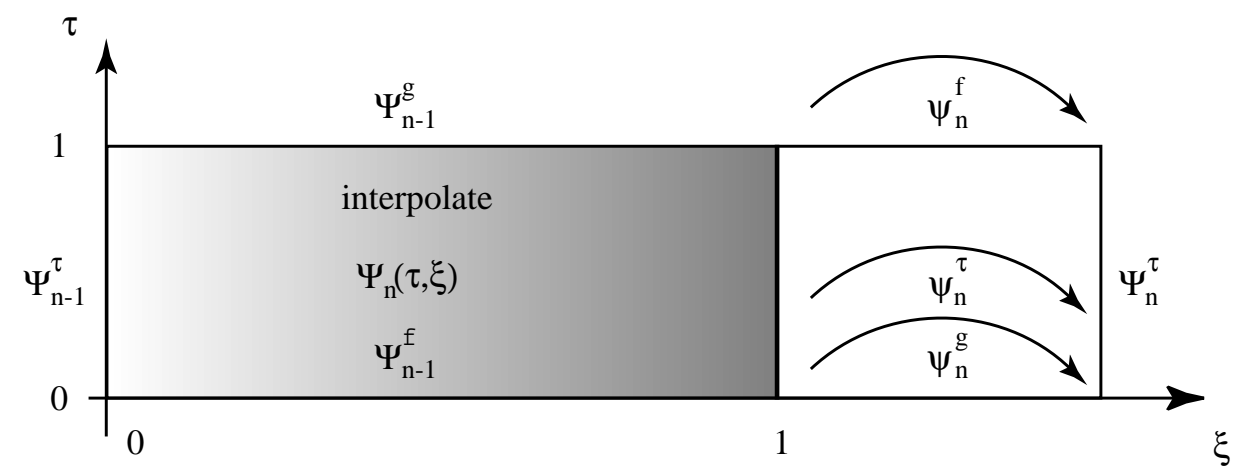

FIGURE 2.2. Constructing interpolating diffeomorphisms.

diffeomorphisms

$$
\Psi_{n, k}^{\tau}:=\Psi\left(\tau, \xi_{k+1}\right) \circ\left(\Psi\left(\tau, \xi_{k}\right)\right)^{-1}
$$

with $\xi_{k}:=4 k /(m-1), k=0, \ldots,(m-5) / 4$, are uniformly close to identity in $\operatorname{Diff}(\Omega)$. They can therefore be realized, at last, by quadruple shootings $\tilde{\psi}_{j}$ as in (2.28). This is the pay-off out of the freedom gained by $n(m-1) / 2$-dimensionally unstable suspension of the global attractors $\mathcal{A}_{f}$ and $\mathcal{A}_{g}$.

Returning to the cumulative shooting maps $\Psi_{n}^{\tau}$, a Morse-Smale homotopy is realized between the suspended attractors $\tilde{\mathcal{A}}_{f}$ and $\tilde{\mathcal{A}}_{g}$ and, consequently, between the original attractors $\mathcal{A}_{f}$ and $\mathcal{A}_{g}$. Therefore, $\mathcal{A}_{f}$ and $\mathcal{A}_{g}$ are indeed globally $C^{0}$ orbit equivalent, and our main theorem will be proved.

\section{Discretization}

As outlined in section 2, we discretize the original semilinear parabolic equation

$$
u_{t}=u_{x x}+f\left(x, u, u_{x}\right) .
$$

In fact, equidistant semidiscretization in $x$ of step size $1 / n$ and the method of lines in $t$-direction yield

$$
\dot{u}_{i}=f_{i}\left(u_{i-1}, u_{i}, u_{i+1}\right),
$$

$i=0, \ldots, n$, with $f_{i}$ given explicitly by

$$
f_{i}\left(u_{i-1}, u_{i}, u_{i+1}\right)=n^{2}\left(u_{i-1}-2 u_{i}+u_{i+1}\right)+f\left(i / n, u_{i}, n\left(u_{i}-u_{i-1}\right)\right) .
$$

Clearly, $u_{i}$ represents $u$ at $x=i / n$. As noted before, Neumann boundary conditions become

$$
u_{-1}:=u_{0}, \quad u_{n+1}:=u_{n} .
$$

We do not advocate (3.2)-(3.4) as a particularly efficient discretization from a numerical point of view. Rather, we prefer this discretization because, for large enough $n$, it preserves

(i) nodal properties of solutions,

(ii) the Morse-Smale structure, 
(iii) the global attractor $\mathcal{A}_{f}$, up to orbit equivalence, and

(iv) the shooting permutation $\pi_{f}$.

Properties (i)-(iii) are essentially known and are surveyed below. Property (iv) will be addressed in section 6 .

Recall that $u:=\left(u_{0}, \ldots, u_{n}\right) \in \mathbb{R}^{n+1}$ will also denote solutions of (3.2), in addition to denoting solutions of (3.1); context will avoid confusion. Properties (i),(ii) hold for general systems (3.2), (3.4), if only $f_{i}$ has positive off-diagonal partial derivatives, alias Jacobi structure, as in (2.16). This is indeed the case for the discretization (3.3) and large $n$, by our dissipation normalization (2.2).

Let the zero number $z(u)$ denote the number of strict sign changes of the sequence of coordinates $u_{0}, \ldots, u_{n}$. Then

$$
t \mapsto z\left(u^{1}(t)-u^{2}(t)\right)
$$

is noncreasing with time $t$, just as in the continuous case (1.21). In fact, $z\left(u^{1}(t)-u^{2}(t)\right)$ drops strictly at $t=t_{0}$ whenever a component $i$ of the nontrivial difference $\eta:=u^{1}-u^{2}$ vanishes and the difference components $i \pm 1$ are of equal sign ("multiple zero"),

$$
\eta_{i}\left(t_{0}\right)=0, \text { and } \eta_{i-1}\left(t_{0}\right) \cdot \eta_{i+1}\left(t_{0}\right) \geq 0,
$$

for some $i=0, \ldots, n$. In case $\eta_{i-1} \cdot \eta_{i+1}>0$ this follows easily from the monotonicity properties of $f_{i}$, which imply

$$
\operatorname{sign} \dot{\eta}_{i}=\operatorname{sign} \eta_{i-1}=\operatorname{sign} \eta_{i+1}
$$

at $t=t_{0}$. For the general case see [MPS90], [FO88]. This settles claim (i).

The Morse-Smale structure (ii) of the finite dimensional system (3.2), (3.4) follows from the dropping properties (3.5), (3.6) of the zero number $z$; see [FO88], [Ang86]. As in the PDE case outlined in the introduction, hyperbolicity of all equilibria automatically entails transversality of their associated stable and unstable manifolds.

The properties (iii) and (iv) rest on the particular nonlinearities $f_{i}$ given by semidiscretization (3.3). First note that (3.2), (3.4) is a dissipative system, in that case. Indeed $\left|u_{i}\right| \geq 2 C_{1}$ or $\left|n\left(u_{i+1}-u_{i}\right)\right| \geq 2 C_{2}$ imply

$$
\dot{u}_{i}=f_{i}\left(u_{i-1}, u_{i}, u_{i+1}\right)=n^{2}\left(u_{i-1}-2 u_{i}+u_{i+1}\right)-u_{i} \text {. }
$$

In particular $\|u\|:=\max \left|u_{i}(t)\right|$ decreases with time, as long as $\|u\| \geq 2 C_{1}$.

By dissipativeness, (3.2)-(3.4) possesses a compact global attractor $\mathcal{A}_{f}^{n}$. Orbit equivalence of $\mathcal{A}_{f}^{n}$ to the global PDE attractor $\mathcal{A}_{f}$ was announced in [Hal94], see p. 24. A proof is in preparation [Rau95]. We indicate our own viewpoint, for convenience. Let $d=\operatorname{dim} \mathcal{A}_{f}=\max \left\{i(u) \mid u \in \mathcal{E}_{f}\right\}$ be the maximal Morse index of any equilibrium $u=u(x) \in \mathcal{E}_{f}$. Define a projection

$$
P: \quad \mathcal{A}_{f} \rightarrow \mathbb{R}^{n+1}
$$

letting $(P u)_{i}:=u(i / n), i=0, \ldots, n$, denote actual values of the $x$-profile $u$. By nodal properties on $\mathcal{A}_{f}$, the projection $P$ is injective if $n+1 \geq d$. In fact, $P$ turns out to be a diffeomorphic embedding of $\mathcal{A}_{f}$ into $\mathbb{R}^{n+1}$ [Mat95]. The main step here is to show that $\mathcal{A}_{f}$ possesses a unique $d$-dimensional tangent plane everywhere, not only on the $d$-dimensional unstable manifolds of its most unstable equilibria, but also, by a limiting procedure, on their closure - which constitutes all of $\mathcal{A}_{f}$. Along the lines of [FR96], section 3, these claims are consequences of the nodal properties (3.5), (3.6) for the flow induced, by linearization, on the Grassmannian of $d$-planes. 
Along any heteroclinic orbit from $u$ to $\tilde{u} \in \mathcal{E}_{f}$, for example, these $d$-planes limit onto the span of the first $d$ eigenfunctions of the target equilibrium $\tilde{u}$. They are then propagated further down in $\mathcal{A}_{f}$, along $W^{u}(\tilde{u})$, as unique tangent planes of a non-unique generalized $d$-dimensional "center"-unstable manifold. At $\tilde{u}$, of course, this generalized center-unstable manifold is tangent to the eigenfunctions of the first $d$ eigenvalues. Propagating along $W^{u}(\tilde{u})$, its tangent spaces remain unique even though the generalized center-manifold is non-unique.

With the embedding $P$ at hand, it is then relatively easy to compare the flow on $\mathcal{A}_{f}$ with the flows on the $d$-dimensional global attractors $\mathcal{A}_{f}^{n}$, for large $n$. Indeed, for $n \rightarrow \infty$, all equilibria become hyperbolic, converging to $\mathcal{E}_{f}$ with eventually constant Morse indices. Their unstable manifolds converge, uniformly in $C^{1}$, and so do the unique $d$-dimensional tangent planes, on the attractors $\mathcal{A}_{f}^{n}$, to the nonunique center-unstable manifolds constructed above. The final observation is $C^{0}$ structural stability of Morse-Smale flows under $C^{1}$-small perturbations. Therefore $\mathcal{A}_{f}$ and $\mathcal{A}_{f}^{n}$ are globally orbit equivalent, for large $n$.

Henceforth, we will fix such a large $n$ and denote $\mathcal{A}_{f}^{n}$ by $\mathcal{A}_{f}$, again. Also the ambiguity of notation in our $u$ seems thoroughly justified, by now.

\section{DisSIPATION}

In this section we discuss conditions for and elementary consequences of dissipativeness, as defined in the introduction, for the continuous PDE case and the (augmented) discrete system.

Recall the dissipation conditions (1.7), (1.8) for the original PDE (1.1), (1.2). Dissipation, together with the parabolic comparison principle alias strong monotonicity (e.g. [Smo83], [Hir88], [Mat86]), implies that (1.1) possesses a maximal and a minimal equilibrium

$$
\bar{u}, \underline{u} \in \mathcal{E}_{f} \subseteq \mathcal{A}_{f}
$$

in the global attractor $\mathcal{A}_{f}$. Indeed $\bar{u}, \underline{u}$ arise, respectively, as $\omega$-limit sets of large positive or negative initial conditions (taken for example $x$-independent). After a linear transformation $u \mapsto \tilde{u}$,

$$
u:=a(x) \tilde{u}+b(x)
$$

we may assume

$$
\bar{u}(x) \equiv+1, \quad \underline{u}(x) \equiv-1,
$$

without loss of generality. Indeed the transformation (4.2) preserves the form of the PDE (1.1). In terms of the original $x$-dependent equilibria $\bar{u}, \underline{u}$ in (4.1), the coefficients $a, b$ in (4.2) read

$$
a=(\bar{u}-\underline{u}) / 2, \quad b=(\bar{u}+\underline{u}) / 2 .
$$

Solutions $\tilde{u}$, like $u$ itself, therefore satisfy Neumann boundary conditions because $a_{x}, b_{x}=0$ at $x=0,1$. We can now assume (4.3) to hold without loss of generality.

Lemma 4.1. Let (4.3) hold and consider $u \in \mathcal{A}_{f}$. Then $|u(x)| \leq 1$ holds for all $x$.

Proof. We argue indirectly, assuming $u\left(x_{0}\right)>1$ without loss of generality. Since $u \in$ $\mathcal{A}_{f}$, the $\alpha$-limit set of $u$ is an equilibrium $u^{-} \in \mathcal{E}_{f}$. Since $z(u-1)$ is nondecreasing, 
in backwards time, and by maximality of $\bar{u} \equiv 1$ in $\mathcal{E}_{f}$, the zero number $z$ of $u^{-}-1$ is nontrivial:

$$
z\left(u^{-}-1\right) \geq 1 .
$$

Now let $u_{0}(x)>u^{-}(x), 1$ be another initial condition. Necessarily $\omega\left(u_{0}\right) \equiv 1$. But because $z$ is nonincreasing, in forward time,

$$
z\left(1-u^{-}\right) \leq z\left(u_{0}-u^{-}\right)=0 .
$$

The contradiction (4.5), (4.6) proves the lemma.

As mentioned in the outline, (2.2), we now replace the dissipation conditions (1.7), (1.8) by the simpler condition

$$
f(x, u, p)=-u,
$$

for $|u| \geq C_{1}$ or $|p| \geq 2 C$. Here $C$ is a constant specified below.

Lemma 4.2. Let $f$ satisfy the dissipation conditions (1.7), (1.8) for $|u| \geq C_{1}>1$, with the normalization (4.3).

Then $|u| \leq 1<C_{1}$ and $\left|u_{x}\right| \leq C$ on the global attractor $\mathcal{A}_{f}$, for some constant C. Moreover, there exists a nonlinearity $\tilde{f}=\tilde{f}(x, u, p) \in C^{2}$ which

(i) coincides with $f$ on $\mathcal{A}_{f}$ and in fact on the larger set where $|u| \leq\left(1+C_{1}\right) / 2$ and $|p| \leq 1.5 C$,

(ii) satisfies (4.7), and

(iii) possesses the same global attractor

$$
\mathcal{A}_{\tilde{f}}=\mathcal{A}_{f},
$$

of course with the same dynamics on it.

Proof. The bound

$$
|u| \leq 1
$$

on $\mathcal{A}_{f}$ was proved in Lemma 4.1. The bound

$$
\left|u_{x}\right| \leq C
$$

follows from compactness of the global attractor $\mathcal{A}_{f} \subset X \subset C^{1}$ under dissipation conditions (1.7), (1.8) on $f$. The gradient bound (4.10) depends on $C_{1}, a, b, \gamma$, but not on $f$. Without loss of generality, we assume $a(u) \geq|u|$ in our construction of C.

We construct $\tilde{f}$ as follows. Let $\mathcal{V} \subset \mathbb{R}^{2}$ be a small open neighborhood of the set where $|u| \geq C_{1}$ or $|p| \geq 2 C$, such that the open dissipation conditions (1.7), (1.8) still hold on $\mathcal{V}$. Let $\tilde{f}:=(1-\tau) f-\tau u$, choosing $0 \leq \tau=\tau(u, p) \leq 1$ with $\tau \equiv 0$ outside $\mathcal{V}$, and $\tau(u, p)=1$ for $|u| \geq C_{1}$ or $|p| \geq 2 C$. Then

(i) $\tilde{f}(x, u, p)=f(x, u, p)$, for $(u, p) \notin \mathcal{V}$,

(ii) $\tilde{f}(x, u, p)=-u$, for $|u| \geq C_{1}$ or $|p| \geq 2 C$, and

(iii) $\tilde{f}$ satisfies (1.7), (1.8) on $\mathcal{V}$.

Note that (iii) holds, with the same $C_{1}, a, b, \gamma$, by convexity of the dissipation conditions (1.7), (1.8) with respect to $f$. Clearly (i), (ii) above imply (i), (ii) in the lemma.

To see that (iii) implies (iii), we first note that $\mathcal{E}_{f}=\mathcal{E}_{\tilde{f}}$. Indeed, in view of the bounds (4.9), (4.10), the nonlinearities $\tilde{f}$ cannot possess an equilibrium $u$ such 
that $\left(u(x), u_{x}(x)\right) \in \mathcal{V}$, for any fixed $x$ or $\tau$. To see that $\mathcal{A}_{f}=\mathcal{A}_{\tilde{f}}$, just recall from (1.20) that $\mathcal{A}_{f}$ is the union of unstable manifolds $W_{f}^{u}(u)$ of $u \in \mathcal{E}_{f}$. Because $|u| \leq 1<C_{1},\left|u_{x}\right| \leq C$ on $\mathcal{A}_{f}$, we conclude $W_{\tilde{f}}^{u}(u)=W_{f}^{u}(u)$ for all $u \in \mathcal{E}_{\tilde{f}}=\mathcal{E}_{f}$. Taking the union over $u \in \mathcal{E}_{\tilde{f}}$, this implies (iii), $\mathcal{A}_{\tilde{f}}=\mathcal{A}_{f}$, and the lemma is proved.

We note that $\pi_{\tilde{f}}=\pi_{f}$, for the associated equilibrium permutations, because $\mathcal{A}_{\tilde{f}}=\mathcal{A}_{f}$ implies $\mathcal{E}_{\tilde{f}}=\mathcal{E}_{f}$. Therefore we can assume (4.7) to hold for $f$ and $g$ in our proof of Theorem 1.1, without loss of generality.

We now consider discrete equations with positive off-diagonal partial derivatives,

$$
\dot{u}_{i}=f_{i}\left(u_{i-1}, u_{i}, u_{i+1}\right),
$$

for $i=0, \ldots, n$, as in $(2.16),(2.17)$. An obviously sufficient condition for dissipativeness of such Jacobi systems is, in general,

$$
u_{i} f_{i}\left(u_{i-1}, u_{i}, u_{i+1}\right)<0
$$

for $\left|u_{i}\right| \geq \max \left\{2 C_{1},\left|u_{i-1}\right|,\left|u_{i+1}\right|\right\}, i=0, \ldots, n$. Indeed, $\|u\|=\max _{i}\left\{\left|u_{i}\right|\right\}$ then decreases monotonically down to level $2 C_{1}$. In our particular case, where $f_{i}$ arises by discretization (3.3), condition (4.7) is obviously satisfied. The global attractor of (4.11) is again called $\mathcal{A}_{f}$, in view of section 3. Our normalization (4.3) still holds, because all $f_{i}( \pm 1, \pm 1, \pm 1)=0$. Therefore Lemma 4.1 carries over to the discrete case.

\section{UNSTABLE SUSPENSION}

In this section we discuss augmented discrete systems

$$
\begin{aligned}
\dot{u}_{i} & =f_{i}\left(u_{i-1 / m}, u_{i}, u_{i+1 / m}\right), \\
\varepsilon \dot{u}_{j} & =u_{j-1 / m}+u_{j+1 / m}
\end{aligned}
$$

as introduced in (2.18). Here $0 \leq i \leq n$ are integer and $\varepsilon>0$. For integer $0<$ $k<m$, the noninteger values $0<j:=i+k / m<n$ indicate the artificially inserted values $u_{j}$ which augment the original system. We impose Neumann boundary conditions (2.19) on the flow of (5.1). Our aim is to recover the global attractor $\mathcal{A}_{f}$ of the original discretized flow

$$
\dot{u}_{i}=f_{i}\left(u_{i-1}, u_{i}, u_{i+1}\right)
$$

with Neumann boundary conditions (2.17). For small $\varepsilon>0$, and up to orbit equivalence, we will find $\mathcal{A}_{f}$ unstably suspended in a center manifold close to the linear subspace

$$
M:=\left\{u_{j-1 / m}+u_{j+1 / m}=0, \text { for all noninteger } 0<j<n\right\} .
$$

This subspace contains all equilibria of the flow (5.1), (2.19) and corresponds to its singular manifold, that is, $M$ is entirely composed of equilibrium points of the formally reduced system for $\varepsilon=0$ in the fast time variable $\tau=t / \varepsilon$. The formally reduced slow flow on $M$, in contrast, is given explicitly by (5.2) with Neumann boundary conditions (2.17), for $\varepsilon=0$. As we will see in the next lemma, it perturbs to a flow with an orbit equivalent compact invariant set on a normally hyperbolic invariant manifold $M_{\varepsilon}$ nearby $M$, for small $\varepsilon>0$. 
Lemma 5.1. Assume $m \equiv 1(\bmod 4), m \geq 5$. Then the discretized flow (5.2), (2.17) is orbit equivalent to the reduced slow flow on the subspace $M$ of dimension $n+1$ by the isomorphism

$$
\begin{aligned}
I: \quad \mathbb{R}^{n+1} & \rightarrow M \subseteq \mathbb{R}^{n m+1}, \\
\left(u_{0}, u_{1}, \ldots, u_{n}\right) & \mapsto\left(u_{0}, u_{1 / m}, \ldots, u_{n}\right)
\end{aligned}
$$

defined by the sign alternation

$$
\begin{aligned}
u_{i+k / m} & =(-1)^{k / 2} u_{i}, \\
u_{i+1-k / m} & =(-1)^{k / 2} u_{i+1},
\end{aligned}
$$

for all $i=0, \ldots, n-1$, and all even $k=2, \ldots, m-1$. In particular

$$
u_{i \pm 1}=u_{i \pm 1 / m}
$$

holds for this embedding, $i=0, \ldots, n$.

The subspace $M$ is uniformly normally hyperbolic for the reduced fast flow. The dimensions of the strong stable and the strong unstable fibers are equal, each given by

$$
n(m-1) / 2 .
$$

For small $\varepsilon>0$, the augmented discrete system (5.1) with Neumann boundary conditions (2.19) possesses a maximal compact invariant set $\tilde{\mathcal{A}}_{f}^{\varepsilon}$, alias its set of bounded solutions, satisfying

$$
\tilde{\mathcal{A}}_{f}^{\varepsilon} \cong I\left(\mathcal{A}_{f}\right) \subseteq M
$$

In particular, $\tilde{\mathcal{A}}_{f}^{\varepsilon}$ is orbit equivalent to $\mathcal{A}_{f}$.

Proof. Because $m \equiv 1(\bmod 4)$, the map $I$ is indeed a well-defined linear isomorphism between $\mathbb{R}^{n+1}$ and $M$. In particular $\operatorname{dim} M=n+1$. Because $m \equiv 1(\bmod$ 4), we may choose $k:=m-1$ even, in (5.5). Because $k / 2$ is still even, this implies (5.6) for $i=1, \ldots, n-1$. For $i=0, n$, equality (5.6) follows from the respective Neumann boundary conditions (2.17), (2.19). Inserting (5.6) into the first equation of the augmented discrete system (5.1) immediately recovers the original discretized flow (5.2), proving flow equivalence with the formally reduced slow system of (5.1), (2.19) for $\varepsilon=0$. We define $\tilde{\mathcal{A}}_{f}^{0}:=I\left(\mathcal{A}_{f}\right) \subseteq M$.

To prove the normal hyperbolicity of $M$ for the reduced fast flow, we follow standard singular perturbation reasoning. For small $\varepsilon>0$ and rescaled fast time $t / \varepsilon$, the fast time transition layers are given approximately by the equations

$$
\dot{u}_{j}=u_{j-1 / m}+u_{j+1 / m},
$$

for all noninteger $0<j<n$ as above. The approximation is of class $C^{\kappa}$, for any $\kappa$. Since $\dot{u}_{i}=0$ for integer $i$, in fast time and for $\varepsilon=0$, the values of $u_{j \pm 1 / m}$ for integer $i=j \pm 1 / m$ in (5.9) are to be considered as constants. Therefore the linearization of (5.9) at any point of $M$, coordinatized by $u_{0}, u_{1}, \ldots, u_{n}$ according to relations (5.5), decouples into $n$ identical copies of equation (5.9). In the first copy, $j$ ranges from $1 / m$ to $(m-1) / m$, only. The constants $u_{i}$, when linearized, provide Dirichlet boundary conditions $u_{0}=u_{1}=0$, and similarly for the other $j$-blocks. Eigenvectors of the right-hand side of (5.9) are therefore given by $u_{j}=\sin (\pi j \ell)$ with associated real, distinct, nonzero eigenvalues

$$
\lambda_{\ell}=2 \cos (\pi \ell / m),
$$


$\ell=1, \ldots, m-1$. Counting $\lambda_{\ell}>0$ and $\lambda_{\ell}<0$ then proves (5.7). Normal hyperbolicity of $M$ follows for the reduced system in the fast time variable and holds uniformly on $M$. Indeed the flow (5.1) is already linear in the $\dot{u}_{j}$ equation. Moreover, the $\dot{u}_{i}$ equation becomes linear for large $\|u\|$, due to definition (3.3) of $f_{i}$ and normalization (4.7) of $f$. Therefore, $M$ is uniformly normally hyperbolic.

For small $\varepsilon>0$, singular perturbation theory implies that the augmented discrete system (5.1), (2.19) has a maximal compact invariant set $\mathcal{A}_{f}^{\varepsilon}$ in a neighborhood $U$ of the compact set $\mathcal{A}_{f}^{0}$; see [Fen79], Theorem 9.1. Near $M \cap U$ the flow (5.1), (2.19) in the fast time variable has a $C^{2}$-smooth $(n+1)$-dimensional center manifold $M_{\varepsilon}$ that depends smoothly on $\varepsilon$ even at $\varepsilon=0$. Restricted to this slow manifold, the slow vector field (5.1), (2.19) becomes a $C^{1}$ regular perturbation problem; see [Fen79] for details. Therefore, by structural stability of the reduced slow Morse-Smale system on $M$, the maximal compact invariant set $\tilde{\mathcal{A}}_{f}^{\varepsilon}$ is orbit equivalent to our original global attractor

$$
\tilde{\mathcal{A}}_{f}^{\varepsilon} \cong I\left(\mathcal{A}_{f}\right)
$$

for small $\varepsilon>0$, and the lemma is proved.

Throughout, we will consider $\varepsilon>0$ fixed and denote $\tilde{\mathcal{A}}_{f}^{\varepsilon}$ by $\tilde{\mathcal{A}}_{f}$.

\section{Discrete SHOOTING}

In this section we study the analogue of the shooting curves $S_{f}^{x}$, introduced in (2.5), for the augmented discrete system (2.17). We make free use of the notation (2.21)-(2.28) for the two-term shooting recursion $\varphi_{j}^{f}, j=0,1 / m, \ldots, n$, the incremental shooting diffeomorphism $\psi_{j}^{f}$ in $\left(u_{j}, v_{j}\right)$-coordinates, the shooting quadruples $\tilde{\psi}_{j}^{f}$, and the cumulative shooting diffeomorphisms $\Psi_{i}^{f}$. We define the discrete ana$\log u e \pi_{f}^{n}$ of the equilibrium shooting permutation $\pi_{f}$. In Lemma 6.1 we show that $\pi_{f}$ does not change under discretization or augmentation. In Lemma 6.2 we highlight the additional freedom gained by augmentation: realization of arbitrary shooting diffeomorphisms near identity by shooting quadruples $\tilde{\psi}_{j}^{f}$.

The shooting permutation $\pi_{f}^{n}$ can be defined for general discrete Jacobi systems

$$
\dot{u}_{i}=f_{i}\left(u_{i-1}, u_{i}, u_{i+1}\right),
$$

$i=0, \ldots, n$, for example with Neumann boundary conditions $u_{-1}=u_{0}, u_{n+1}=$ $u_{n}$. Assume the equilibrium set $\left\{u^{1}, \ldots, u^{N}\right\} \subseteq \mathbb{R}^{n+1}$ is finite. Just as in the continuous case, the ordering at $i=0$,

$$
u_{0}^{1}<\ldots<u_{0}^{N},
$$

defines a permutation $\pi=\pi_{f}^{n}$ by the ordering of the values

$$
u_{n}^{\pi(1)}<\ldots<u_{n}^{\pi(N)}
$$

at the other Neumann boundary $i=n$.

The discrete analogue of the shooting curves $S_{f}^{x}$ are the $C^{1}$ Jordan curves

$$
S_{i}^{f}:=\Psi_{i}^{f}(\operatorname{diag}) \subseteq \mathbb{R}^{2} .
$$

Here $\Psi_{i}^{f}$ is the cumulative shooting diffeomorphism, and diag $=\left\{u_{-1}=v_{-1}\right\}$ denotes the diagonal in $\mathbb{R}^{2}$. As in the continuous case, the permutation $\pi_{f}^{n}$ can also 
be viewed as relating the two possible orderings of the intersection points

$$
S_{n}^{f} \cap \operatorname{diag},
$$

along the diagonal $\operatorname{diag}=\left\{u_{n}=v_{n}\right\}$, and along $S_{n}^{f}$, respectively. We note that transverse crossings, in (6.5), indicate hyperbolic equilibria of system (6.1), similarly to the continuous case.

Let $\pi_{f}$ denote the original permutation associated to the equilibria $\mathcal{E}_{f}$, let $\pi_{f}^{n}$ denote the permutation associated to the discretization (3.2)-(3.4), and finally $\tilde{\pi}_{f}^{m n}$ the permutation associated to the augmented system (2.18)-(2.19).

Lemma 6.1. Let $n$ be large enough and $m \equiv 1(\bmod 4), m \geq 1$. Then

$$
\pi_{f}=\pi_{f}^{n}=\tilde{\pi}_{f}^{m n} .
$$

Proof. The convergence of discretized equilibria is uniform in $x$, for $n \rightarrow \infty$, if we interpolate linearly between the values $u_{i}$ at $x=i / n$. More precisely, uniform convergence holds on bounded subsets of $\left(u_{i}, v_{i}\right)$. The normalization $f=-u$ for large $|u|,|p|$ given in (2.2) and section 5 avoids the appearance of additional "spurious" equilibria in the discretized equations which otherwise might escape to infinity for $n \rightarrow \infty$. This proves $\pi_{f}=\pi_{f}^{n}$, by definitions (1.13)-(1.14) and (6.2)-(6.3).

Since the augmentation (2.17), (2.18) does not change the cumulative shooting diffeomorphism $\Psi_{f}^{n}$, at all, the claim $\pi_{f}^{n}=\tilde{\pi}_{f}^{m n}$ is obvious. This proves the lemma.

Encouraged by Lemma 6.1, we simply denote all three permutations in (6.6) by $\pi_{f}$, from now on.

We turn to realization of near identity diffeomorphisms by shooting quadruples

$$
\tilde{\psi}_{j}=\psi_{j+3 / m} \circ \ldots \circ \psi_{j},
$$

for noninteger $j, \ldots, j+3 / m$. The unperturbed incremental shooting diffeomorphisms

$$
\psi_{j+k / m}=R,
$$

$k=0, \ldots, 3$, are pure rotations by 90 degrees, independently of the original nonlinearity $f$. In particular, $\tilde{\psi}_{j}=i d$ in the unperturbed case. To preserve the Jacobi structure of the augmented system (2.17), under perturbation, we require the incremental shooting diffeomorphism

$$
\psi_{j^{\prime}+1 / m}\left(u_{j^{\prime}}, v_{j^{\prime}}\right)=\left(v_{j^{\prime}}, \varphi_{j^{\prime}+1 / m}\left(u_{j^{\prime}}, v_{j^{\prime}}\right)\right),
$$

$j^{\prime}=j+(k-1) / m$, to exhibit uniformly negative partial derivatives $\partial_{u_{j^{\prime}}} \varphi_{j^{\prime}+1 / m}$. Note that the unperturbed case is

$$
\varphi_{j^{\prime}+1 / m}\left(u_{j^{\prime}}, v_{j^{\prime}}\right)=-u_{j^{\prime}} .
$$

Lemma 6.2. Let $\tilde{\psi}_{j}$, for noninteger $j$, be any diffeomorphism of $\mathbb{R}^{2}$ with Jacobian uniformly $\delta$-close to identity, for some $0<\delta<1 / 2$.

Then there exist four mappings $\varphi_{j}, \ldots, \varphi_{j+3 / m}$ as in (6.9), such that their composition quadruple (6.7) realizes the prescribed diffeomorphism $\tilde{\psi}_{j}$.

More explicitly, the partial derivatives of $\varphi_{j}, \ldots, \varphi_{j+3 / m}$ can be chosen $\frac{\delta}{1-\delta}$-close to those of the unperturbed case (6.10). 
Proof. Without loss of generality, let $j^{\prime}=0, \ldots, 3 / \mathrm{m}$. Simplifying notation, in this proof we write $\tilde{\psi}$, for $\tilde{\psi}_{j}$, replacing $j^{\prime}$ by $0,1,2,3$, and $j=j^{\prime}+1$ by $1,2,3,4$. We then have to realize

$$
\tilde{\psi}=\psi_{4} \circ \psi_{3} \circ \psi_{2} \circ \psi_{1}
$$

near identity. In fact, we do not perturb

$$
\psi_{2}=\psi_{1}=R
$$

Therefore it suffices to prove that $\psi_{4} \circ \psi_{3}$ can realize any diffeomorphism $-\tilde{\psi}$ which is $\delta$-close to $-i d$ in $C^{1}$.

In terms of $\varphi_{4}, \varphi_{3}$, we can rewrite $\psi_{4} \circ \psi_{3}$ as

$$
\left(\psi_{4} \circ \psi_{3}\right)(u, v)=\left(\varphi_{3}(u, v), \varphi_{4}\left(v, \varphi_{3}(u, v)\right)\right) .
$$

This defines $\varphi_{3}, \psi_{3}$ in terms of the given diffeomorphism $-\tilde{\psi}$, trivially. We note two obvious estimates for the partial derivatives $\partial_{1} \varphi_{3}, \partial_{2} \varphi_{3}$ :

$$
\begin{aligned}
\left|1+\partial_{1} \varphi_{3}\right| & \leq \delta, \\
\left|\partial_{2} \varphi_{3}\right| & \leq \delta .
\end{aligned}
$$

In particular $\partial_{1} \varphi_{3}<0$ holds uniformly, if we choose $\delta<1$. Moreover, $\varphi_{3}$ is $\delta$-close to the unperturbed case.

Let $\varphi(u, v)$ denote the second component of the prescribed diffeomorphism $-\tilde{\psi}$,

$$
\begin{aligned}
\left|\partial_{1} \varphi\right| & \leq \delta, \\
\left|1+\partial_{2} \varphi\right| & \leq \delta .
\end{aligned}
$$

To realize $\psi_{4} \circ \psi_{3}=-\tilde{\psi}$, we simply have to define

$$
\varphi_{4}:=\varphi \circ \psi_{3}^{-1} \text {. }
$$

With this definition, we now partially differentiate

$$
\varphi_{4}\left(v, \varphi_{3}(u, v)\right)=\varphi(u, v),
$$

with respect to $u$ and $v$, to obtain

$$
\begin{aligned}
\partial_{2} \varphi_{4} \cdot \partial_{1} \varphi_{3} & =\partial_{1} \varphi \\
\partial_{1} \varphi_{4}+\partial_{2} \varphi_{4} \cdot \partial_{2} \varphi_{3} & =\partial_{2} \varphi .
\end{aligned}
$$

We have to show $\partial_{1} \varphi_{4}<0$, uniformly. Inserting estimates (6.14), (6.15) into the first equation of (6.18), we immediately see

$$
\left|\partial_{2} \varphi_{4}\right|=\left|\partial_{1} \varphi\right| /\left|\partial_{1} \varphi_{3}\right| \leq \delta /(1-\delta) .
$$

Reinserting (6.19) into the second equation of (6.18) we find

$$
\left|1+\partial_{1} \varphi_{4}\right| \leq\left|1+\partial_{2} \varphi\right|+\left|\partial_{2} \varphi_{4} \cdot \partial_{2} \varphi_{3}\right| \leq \delta+\frac{\delta}{1-\delta} \cdot \delta=\delta /(1-\delta) .
$$

Hence $\partial_{1} \varphi_{4}<0$, uniformly, provided we fix $\delta<1 / 2$. Moreover all $\left(\partial_{1} \varphi_{j}, \partial_{2} \varphi_{j}\right)$ are $\frac{\delta}{1-\delta}$-close to the unperturbed case. 


\section{PATHS AND LOOPS OF DIFFEOMORPHISMS}

This section contains details of the construction of our final, non-naive MorseSmale homotopy in our class of augmented discretized systems (2.18). For an outline and notation see (2.29)-(2.34). We first define the planar region $\Omega$, outside of which the nonlinearities $f$ and $g$ produce identical incremental and cumulative shooting diffeomorphisms. We then review the explicit construction of the prescribed homotopies $\Psi_{i}^{\tau}, i=1, \ldots, n$. Facts concerning their relation to $\Omega$ are collected in (7.1)-(7.8). In Lemma 7.1 we state the property $\pi_{1}(\operatorname{Diff}(\Omega))=0$, which is used for the construction of the interpolating cumulative shootings $\Psi(\tau, \xi)$, in Lemma 7.2. We conclude with a final discretization of $\xi$, which realizes the homotopy of cumulative shootings in our augmented discretized system (2.18).

We construct the disc $\Omega \subset \mathbb{R}^{2}$ as follows. Consider the original iterations $\left(u_{i}, v_{i}\right)$ for equilibria of the discretized system (2.16) or, equivalently, for the unperturbed, augmented discretization (2.18); see (2.25). For $i=0, \ldots, n-1$ let

$$
\Omega_{i}^{f}:=\left\{\left(u_{-1}, v_{-1}\right) \in \mathbb{R}^{2}|| u_{i} \mid \leq 2 C_{1} \text { and } n\left|u_{i}-v_{i}\right| \leq 2 C_{2}\right\}
$$

The constants $C_{1}, C_{2}$ were used for normalization of $f, g$, in (2.2), and the superscript $f$ emphasizes $f$-dependence of the iteration. Denote

$$
\Omega^{f}:=\bigcup_{i=0}^{n-1} \Omega_{i}^{f}
$$

and let $\Omega$ be any closed planar disc such that

$$
\Omega \supseteq \Omega^{f} \cup \Omega^{g} \text {. }
$$

Consider any initial condition $\left(u_{-1}, v_{-1}\right) \notin \Omega$. Then the $f$ - and $g$-trajectories of $\left(u_{-1}, v_{-1}\right)$ coincide, by normalization. In particular

$$
\begin{aligned}
\varphi_{i}^{f} & =\varphi_{i}^{g}, \\
\psi_{i}^{f} & =\psi_{i}^{g}, \\
\Psi_{i}^{f} & =\Psi_{i}^{g}, \\
S_{i}^{f} & =S_{i}^{g}
\end{aligned}
$$

holds outside $\Omega$, and on $\partial \Omega$, for all $i=0, \ldots, n$; see $(2.24),(2.25),(2.29),(6.4)$ for notation. The equality of the shooting curves "outside $\Omega$ " is meant to hold for corresponding initial conditions $u_{-1}=v_{-1}$ outside $\Omega$, of course.

For $i=0, \ldots, n-1$ we will now fall back on our original, naive idea of a standard homotopy for $\varphi_{i}, \psi_{i}$. Only for the iteration from $i=n-1$ to $i=n$ we will make use of the additional flexibility procured by augmentation. So, for $i=0, \ldots, n-1$ and $0 \leq \tau \leq 1$ define

$$
\begin{aligned}
\varphi_{i}^{\tau} & :=(1-\tau) \varphi_{i}^{f}+\tau \varphi_{i}^{g}, \\
\psi_{i}^{\tau} & :=(1-\tau) \psi_{i}^{f}+\tau \psi_{i}^{g},
\end{aligned}
$$

and let the cumulative shootings $\Psi_{i}^{\tau}$ be defined via composition of $\psi_{i}^{\tau}$, as before. Similarly, we obtain shooting curves $S_{i}^{\tau}, \psi_{i}^{\tau}$, as before. By definition of $\Omega$, equations (7.4) expand to

$$
\begin{aligned}
\varphi_{i}^{f} & =\varphi_{i}^{\tau}=\varphi_{i}^{g}, \\
\psi_{i}^{f} & =\psi_{i}^{\tau}=\psi_{i}^{g}, \\
\Psi_{i}^{f} & =\Psi_{i}^{\tau}=\Psi_{i}^{g}, \\
S_{i}^{f} & =S_{i}^{\tau}=S_{i}^{g}
\end{aligned}
$$

outside $\Omega$. 
For the final step, $i=n$, such a naive homotopy is of course prohibitive because it does not preserve hyperbolicity. Instead we prescribe some planar diffeotopy $\Phi^{\tau}$, in the spirit of (2.12), from $\Phi^{0}=\Psi_{n}^{f}$ to $\Phi^{1}=\Psi_{n}^{g}$, such that transversality of the shooting curves to the diagonal is preserved throughout the diffeotopy:

$$
\Phi^{\tau}(\operatorname{diag}) \pi \operatorname{diag}
$$

for all $0 \leq \tau \leq 1$. Such a planar diffeotopy exists because $\pi_{f}=\pi_{g}$ for the shooting permutations of $S_{n}^{f}=\Phi^{0}(\operatorname{diag})$ and of $S_{n}^{g}=\Phi^{1}(\operatorname{diag})$. By normalization of $f, g$ outside $\Omega$, we may assume

$$
\Psi_{n}^{f}=\Phi^{\tau}=\Psi_{n}^{g}
$$

there. The crucial task of this section is, now, to realize the prescribed homotopy $\Phi^{\tau}$ by appropriate perturbations of the $m-1$ incremental shootings $\psi_{j}, j=n-$ $1+1 / m, \ldots, n-1 / m$, such that

$$
S_{n}^{\tau}=\Psi_{n}^{\tau}(\operatorname{diag})=\Phi^{\tau}(\operatorname{diag})
$$

holds for the cumulative shootings $\Psi_{n}^{\tau}$, in analogy to (2.13). The construction of $\Psi_{n}^{\tau}$ is prepared in the following two lemmas.

Recall that $\operatorname{Diff}_{*}(\Omega)$ denotes the space of all diffeomorphisms of the planar disc $\Omega$ which fix the boundary $\partial \Omega$ pointwise. The topology on $\operatorname{Diff}_{*}(\Omega)$ is the $C^{1}$-topology. As before, $\pi_{1}$ denotes the fundamental group.

\section{Lemma 7.1.}

$$
\pi_{1}\left(\operatorname{Diff}_{*}(\Omega)\right)=0 .
$$

Proof. This is Smale's theorem; see [Cer68], appendix.

We can now construct the two-parameter interpolation $\Psi(\tau, \xi)$ mentioned in (2.32), for $i=n$. We have slightly changed notation: for clarity, the prescribed path $\Psi_{n}^{\tau}$ of diffeomorphisms is distinguished as $\Phi^{\tau}$, here, whereas the notation $\Psi_{n}^{\tau}$ is strictly reserved for cumulative shootings which are realized by perturbations of augmented discrete systems in the sense of (2.18), (2.19).

Lemma 7.2. In the above setting, there exists a continuous two-parameter family $\Psi=\Psi(\tau, \xi), 0 \leq \tau, \xi \leq 1$, of planar diffeomorphisms with the following properties (see Figure 2.2 and (2.32). Let $\psi_{n}^{\tau}:=(1-\tau) \psi_{n}^{f}+\tau \psi_{n}^{g}$. Then, for all $0 \leq \tau, \xi \leq 1$,

$$
\begin{aligned}
& \Psi(\tau, \xi=0)=\Psi_{n-1}^{\tau}, \\
& \Psi(\tau, \xi=1)=\left(\psi_{n}^{\tau}\right)^{-1} \Phi^{\tau}, \\
& \Psi(\tau=0, \xi)=\Psi_{n-1}^{\tau=0}, \\
& \Psi(\tau=1, \xi)=\Psi_{n-1}^{\tau=1} .
\end{aligned}
$$

Specifically outside $\Omega$, we have

$$
\Psi(\tau, \xi)=\Psi_{n-1}^{\tau} .
$$

Proof. Outside $\Omega$, the definition (7.12) of $\Psi(\tau, \xi)$ fulfills all the requirements of (7.11). Indeed, the second equality follows from (7.8) and the definitions of $\psi_{n}, \Psi_{n}$. The remaining equalities hold trivially.

We now construct $\Psi(\tau, \xi)$ inside $\Omega$. Let $\tilde{\Psi}(\tau, \xi)$ be a contraction, in $\operatorname{Diff}_{*}(\Omega)$, of the loop

$$
\tau \mapsto \tilde{\Psi}(\tau, \xi=1):=\left(\psi_{n}^{\tau} \circ \Psi_{n-1}^{\tau}\right)^{-1} \Phi^{\tau},
$$


$0 \leq \tau \leq 1$. In fact, $\tilde{\Psi}(\tau, 1)=i d$ for $\tau=0,1$, and, outside $\Omega$, for $0 \leq \tau \leq 1$; see (2.29), (7.5), (7.6), (7.8). Therefore $\tilde{\Psi}(\tau, \xi) \in \operatorname{Diff}_{*}(\Omega)$ indeed exists, continuously in $(\tau, \xi)$, by triviality of the fundamental group as observed in Lemma 7.1. Note that

$$
\tilde{\Psi}(0, \xi)=\tilde{\Psi}(1, \xi)=\tilde{\Psi}(\tau, 0)=i d,
$$

throughout $0 \leq \tau, \xi \leq 1$. With the help of $\tilde{\Psi}$ we can now easily construct $\Psi$ as

$$
\Psi(\tau, \xi):=\Psi_{n-1}^{\tau} \circ \tilde{\Psi}(\tau, \xi) .
$$

Then the second equation of (7.11) follows from (7.15) and (7.13). The remaining three equations of (7.11) follow from (7.15) and (7.14). This completes the construction inside $\Omega$.

A remaining issue is the $C^{1}$-matching of the diffeomorphisms $\Psi(\tau, \xi)$ along $\partial \Omega$. Pulling this question back to $\tilde{\Psi}(\tau, \xi)$, via (7.15), it is sufficient to provide diffeomorphisms which are identity in a small neighborhood of $\partial \Omega$ in $\Omega$. This is easy to achieve, by first performing the above construction in a slightly smaller disc $\Omega^{\prime} \subset \Omega$, and subsequently smoothing out the transition in a small annulus around $\partial \Omega^{\prime}$ in $\Omega$. This completes the proof of Lemma 7.2.

In the remaining section we conclude our proof of Theorem 1.1, specifying the intermediate incremental discretization steps $\psi_{j}$ with noninteger $0<j<n$, and summarizing the Morse-Smale homotopy from $f$ to $g$.

For noninteger $0<j<n-1$, we do not perturb $\psi_{j}$ away from rotation by 90 degrees. In fact, we could do without any augmentations between $i=0$ and $i=n-1$. This follows because the homotopies $\psi_{i}^{\tau}, i=0, \cdots, n-1$, are all realized within the class of maps $\varphi_{i}^{\tau}(u, v)$ with uniformly negative partial derivatives $\partial_{u} \varphi_{i}^{\tau}$. Standard homotopy $(1-\tau) \varphi_{i}^{f}+\tau \varphi_{i}^{g}$ does the job. We have nevertheless inserted all these artificial discretization points, just for notational convenience.

It is for noninteger $n-1<j<n$, where augmentation seems really indispensable. Following (2.34), we choose $m \equiv 1(\bmod 4)$ large enough such that each of the $(m-1) / 4$ diffeomorphisms

$$
\Psi_{n, k}^{\tau}:=\Psi\left(\tau, \xi_{k+1}\right) \circ\left(\Psi\left(\tau, \xi_{k}\right)\right)^{-1}
$$

is $\delta$-close to identity in $C^{1}$-norm. Here $\xi_{k}=4 k /(m-1), k=0, \ldots,(m-5) / 4,0 \leq$ $\tau \leq 1, \delta<1$, and $\Psi(\tau, \xi)$ is chosen as in Lemma 7.2. By Lemma 6.2, each of the diffeomorphisms can be realized as a shooting quadruple

$$
\Psi_{n, k}^{\tau}=\psi_{n-1+(4 k+4) / m}^{\tau} \circ \cdots \circ \psi_{n-1+(4 k+1) / m}^{\tau}
$$

with incremental shooting maps $\psi_{j}, \varphi_{j}$ respecting the partial derivative condition of Jacobi systems. Only for $\tau=0,1$, the incremental shootings are rotations by 90 degrees; for in between $\tau$ they are perturbed. With this choice of homotopy, the cumulative shooting $\Psi_{n}^{\tau}$ realizes the prescribed diffeotopy $\varphi^{\tau}$ of discrete shooting curves,

$$
\Psi_{n}^{\tau}=\Phi^{\tau}
$$


for $0 \leq \tau \leq 1$, as required essentially in (2.13). Indeed, (7.11), (7.16), and (7.17) imply

$$
\begin{aligned}
\Psi_{n}^{\tau} & =\psi_{n}^{\tau} \circ \psi_{n-1+(m-1) / m}^{\tau} \circ \cdots \circ \psi_{n-1+1 / m}^{\tau} \circ \Psi_{n-1}^{\tau} \\
& =\psi_{n}^{\tau} \circ \Psi_{n,(m-5) / 4}^{\tau} \circ \cdots \circ \Psi_{n, 0}^{\tau} \circ \Psi_{n-1}^{\tau} \\
& =\psi_{n}^{\tau} \circ \Psi\left(\tau, \xi_{(m-1) / 4}\right) \circ \Psi(\tau, 0)^{-1} \circ \Psi_{n-1}^{\tau} \\
& =\psi_{n}^{\tau} \circ \Psi(\tau, 1) \\
& =\Phi^{\tau}
\end{aligned}
$$

which proves (7.18). This completes our construction of a hyperbolicity preserving homotopy of shooting curves from $S_{n}^{f}$ to $S_{n}^{g}$, in an augmented discrete context.

However, there still remains a last homotopy step. By (7.19), we have constructed a shooting homotopy, for all solutions of iterations

$$
0=f_{j}\left(u_{j-1 / m}, u_{j}, u_{j+1 / m}\right)
$$

with given $u_{0}, u_{-1 / m}$. We have not yet constructed a Morse-Smale homotopy for the augmented discrete systems

$$
\dot{u}_{j}=f_{j}\left(u_{j-1 / m}, u_{j}, u_{j+1 / m}\right),
$$

under Neumann boundary conditions.

To complete this final step, consider nonlinearities $f_{j}, \tilde{f}_{j}$ which are both dissipative, as in (4.12), and in addition induce the same iteration via (7.20), that is,

$$
\varphi_{j}=\tilde{\varphi}_{j}
$$

for $\tilde{\varphi}_{j}:=\varphi_{j}^{\tilde{f}}$ and all $j$. Here we think of

$$
\tilde{f}_{j}\left(u_{j-1 / m}, u_{j}, u_{j+1 / m}\right):=u_{j+1 / m}-\tilde{\varphi}_{j}\left(u_{j-1 / m}, u_{j}\right) .
$$

Note that $\operatorname{sign} \tilde{f}_{j}=\operatorname{sign} f_{j}$, and hence $\tilde{f}_{j}$ satisfies the same dissipation condition (4.12) as does $f_{j}$ itself. We show that

$$
\mathcal{A}_{f} \cong \mathcal{A}_{\tilde{f}}
$$

holds for the corresponding maximal compact invariant sets (due to hyperbolic suspension, they are not attractors any more). Indeed, a standard homotopy $(1-\tau) f_{j}+\tau \tilde{f}_{j}$ works: none of the iterations $\varphi_{j}, \psi_{j}, \Psi_{j}$, or finally the shooting curves $S_{n}^{f}$ changes at all during this homotopy. In particular, the homotopy is automatically Morse-Smale. This implies (7.24). Using the same argument on $g, \tilde{g}$, we summarize

$$
\mathcal{A}_{f} \cong \mathcal{A}_{\tilde{f}} \cong \mathcal{A}_{\tilde{g}} \cong \mathcal{A}_{g}
$$

The first and last orbit equivalence are stated in (7.24). The central orbit equivalence follows from (7.18), (7.19), because via construction (7.23) our hyperbolicity preserving homotopy $\varphi_{j}^{\tau}$ induces a Morse-Smale homotopy $\tilde{f}_{j}^{\tau}$ from $\tilde{f}$ to $\tilde{g}$. This proves (7.25) and Theorem 1.1. 


\section{DisCusSion}

Equipped with the proof of our main result, Theorem 1.1, we collect some criticism, generalizations, and open questions. We begin with a discussion of dissipation conditions, narrowing our view to $f=f(x, u)$ and also widening it to more general semilinear and quasilinear PDEs. Keeping the space variable $x$ one-dimensional, we address other separated as well as periodic boundary conditions. The latter case is basically as open as the case of monotone feedback delay equations. For dissipative Jacobi systems, not necessarily arising by space semidiscretization, we announce a result on global orbit equivalence of global attractors in analogy to Theorem 1.1; see Theorem 8.1. It turns out that, from our global attractor point of view, the class of all dissipative Jacobi systems is not richer than the class of all dissipative PDEs (1.1). The question of the mininal dimension $n+1$ of a Jacobi system which realizes a given global PDE attractor of dimension $d$ remains open, however. We conclude with a discussion of the relation between connection equivalence and orbit equivalence of global attractors, and with the problem of a geometric, simplicial description of global attractors.

We recall our dissipation conditions (1.7), (1.8) which state that

$$
\begin{aligned}
f(x, u, 0) \cdot u & <0, \\
|f(x, u, p)| & <a(u)+b(u)|p|^{\gamma}
\end{aligned}
$$

for large $|u|+|p|$. These conditions are by no means necessary. For example, the second condition can be replaced with the crude unilateral constraint $\left(f_{x}+f_{u} p\right) p<$ 0 . We have used (8.1) in Lemma 4.2 in order to normalize

$$
f(x, u, p)=-u
$$

for large $|u|+|p|$. Actually, only convexity of (8.1) with respect to $f$ was used. More generally, any cut-off which replaces a general, dissipative $f$ by an $f$ satisfying (8.2), while preserving the global attractor, would enable us to assume (8.2) without loss of generality. Such a cut-off replacement can be achieved in the class of abstract nonlinearities $f: X^{\alpha} \rightarrow X$, where $X^{\alpha}$ denotes a fractional power space related to diffusion. See for example [MPS88]. But our present proof requires a cut-off $f=f(x, u, p)$ which preserves, for example, the shooting description of equilibria and still does not enlarge the global attractor $\mathcal{A}_{f}$, the equilibrium set $\mathcal{E}_{f}$, or the shooting permutation $\pi_{f}$.

Generalizations to space dependent diffusion terms

$$
u_{t}=c(x) u_{x x}+f\left(x, u, u_{x}\right)
$$

with uniformly positive $c(x)$ can be achieved easily, for example, by a reparametrization of $x$. The fully nonlinear, but still uniformly parabolic and dissipative case

$$
u_{t}=f\left(x, u, u_{x}, u_{x x}\right)
$$

is not sufficiently investigated on a technical level, at present, to apply our ideas directly. We still expect global attractors to be characterized by shooting permutations, up to orbit equivalence. If this is the case, we argue below that these "more nonlinear" equations will not generate additional Morse-Smale attractor types beyond the semilinear case.

The maximal compact invariant set $\mathcal{A}_{f}$ can also be associated to non-dissipative nonlinearities $f$. This observation applies to equations with finite time blow up and, mutatis mutandis, to degenerate diffusion problems of porous media type. For a 
recent survey see [SGKM95]. In principle, our approach via shooting permutations should be applicable to the structure of $\mathcal{A}_{f}$ and might, in conjunction with nodal properties, even lead to qualitative information on some blow up trajectories. To our knowledge, this direction has not been followed in the existing literature.

Natural separated boundary conditions of mixed type can be written as

$$
\begin{array}{lll}
(1-\tau) u-\tau u_{x}=0 & \text { at } & x=0, \\
(1-\tau) u+\tau u_{x}=0 & \text { at } & x=1
\end{array}
$$

with $0 \leq \tau \leq 1$. We have treated Neumann boundary conditions, $\tau=1$, so far. The Dirichlet case, $\tau=0$, as well as the remaining mixed cases $0<\tau<1$ can be treated along the same lines. We just redefine the (oriented) shooting curves $S_{f}^{x}$ as $x=1$ images of the boundary condition (8.5) at $x=0$, under $x$-shooting. Similarly, we adapt the shooting permutation $\pi_{f}$ to encode the discrepancy of orderings of equilibria along the (oriented) boundary condition line (8.5), at $x=1$, and along the shooting curve $S_{f}^{x=1}$, respectively. Imposing suitable dissipation conditions for $f$, Theorem 1.1 remains valid:

$$
\pi_{f}=\pi_{g} \quad \Rightarrow \quad \mathcal{A}_{f} \cong \mathcal{A}_{g} .
$$

Perhaps more surprisingly, all attractors already arise in the Neumann case treated here, up to orbit equivalence. New structurally stable attractor types do not arise, for any $0 \leq \tau<1$. For more details see [Fie96]. Adaptations to mildly nonlinear boundary conditions seem feasible.

The case of periodic boundary conditions, $x \in S^{1}$, is inherently more difficult. Although a Morse-Smale structure persists, the variational structure is lost. In fact, nontrivially time periodic solutions appear, for $f=f(u, p)$ [AF88]. Even in the gradient case $f=f(x, u)$, where the Lyapunov functional (1.17), (1.18) works, we do not possess an analogue of the shooting permutation $\pi_{f}$. Of course, the braid type of the equilibrium profiles in $(x, u, p)$-space comes to mind.

A particularly interesting example from differential geometry is the shortening of closed curves, proportionally to their curvature, on surfaces. For surveys, including intersection properties like the zero number, see [Ang90], [Ang91]. Two difficulties are combined: finite time blow-up and $S^{1}$-structure. Asymptotic states are closed geodesics. Globally defined solutions correspond to heteroclinics between closed geodesics. Blow-up greatly complicates the topology of a phase space $X$ of this infinite-dimensional dynamical system. The $S^{1}$-structure, on the other hand, prevents us from isolating a characterizing discrete object, like our permutation $\pi$, for the set of globally defined solutions of curve shortening.

Theorem 1.1 was proved via discretization

$$
\dot{u}_{i}=f_{i}\left(u_{i-1}, u_{i}, u_{i+1}\right),
$$

$i=0, \ldots, n$, subsequent augmentation, and a Morse-Smale homotopy. Skipping just the initial discretization step, we immediately obtain the analogous result for Jacobi systems (8.7) provided $f_{i}$ is also dissipative, as stated in (4.12). We state this result next. Again $\mathcal{A}_{f}$ denotes the global attractor of (8.7) for $f=\left(f_{0}, \ldots, f_{n}\right)$ and under Neumann boundary conditions $u_{-1}=u_{0}, u_{n+1}=u_{n}$. The permutation $\pi_{f}$ was defined in (6.2), (6.3) for hyperbolic sets $\mathcal{E}_{f}$ of equilibria.

Theorem 8.1. Consider Jacobi systems (8.7). Let $f, g \in C^{1}$ satisfy dissipation condition (4.12). Assume $\mathcal{E}_{f}, \mathcal{E}_{g}$ are hyperbolic. Then $\pi_{f}=\pi_{g}$ implies $\mathcal{A}_{f} \cong \mathcal{A}_{g}$.

Proof. See sections 2, 4-7. 
Let $\mathcal{A}^{\text {dis }}$ denote the set of all global attractors of spatially discrete dissipative Jacobi systems (8.7) as in Theorem 8.1, up to orbit equivalence. We do not require (8.7) to arise by discretization. Similarly, let $\mathcal{A}^{\text {con }}$ denote the same set for spatially continuous PDEs (1.1) as in Theorem 1.1. By discretization, section 3, we know $\mathcal{A}^{\text {con }} \subseteq \mathcal{A}^{\text {dis }}$.

Theorem 8.2. In the above setting,

$$
\mathcal{A}^{\text {con }}=\mathcal{A}^{\text {dis }} .
$$

Proof. We show how (8.8) follows from further results in three related papers.

It remains to show $\mathcal{A}^{\text {con }} \supseteq \mathcal{A}^{\text {dis }}$ : the class (8.7) of spatially discrete Jacobi systems is not richer than the class (3.2), (3.3) of spatially discretized systems. Consider any permutation $\pi_{f}$, for (8.7). From the permutation $\pi_{f}$, the Morse indices $i\left(u^{k}\right) \geq 0$ of equilibria $u^{k} \in \mathcal{E}_{f}, k=1, \ldots, N$, can be determined explicitly:

$$
i\left(u^{k}\right)=\sum_{l=1}^{k-1}(-1)^{l+1} \operatorname{sign}\left(\pi_{f}^{-1}(l+1)-\pi_{f}^{-1}(l)\right) .
$$

Here $i\left(u^{1}\right)=i\left(u^{N}\right)=0$, by dissipation. See [FR97] for a proof and see also [Roc94]. Perhaps not surprisingly, in view of discretization, the same formula is valid in the spatially continuous case (see [FR96]). Moreover it turns out that any such permutation $\pi_{f}$ also arises as

$$
\pi_{\tilde{f}}=\pi_{f}
$$

for a suitable dissipative nonlinearity $\tilde{f}$, in the spatially continuous case, see [FR98]. As in section 3 , let $\mathcal{A}_{\tilde{f}}^{n}$ denote a suitable discretization of $\mathcal{A}_{\tilde{f}}$ such that

$$
\mathcal{A}_{\tilde{f}} \cong \mathcal{A}_{\tilde{f}}^{n} .
$$

For $n$ large enough we also conclude $\pi_{\tilde{f}}=\pi_{\tilde{f}}^{n}$; see Lemma 6.1. Combined with (8.10) this implies $\pi_{\tilde{f}}^{n}=\pi_{\tilde{f}}=\pi_{f}$. By Theorem 8.1 we obtain

$$
\mathcal{A}_{\tilde{f}}^{n} \cong \mathcal{A}_{f},
$$

for these spatially discrete attractors. Together, (8.11) and (8.12) prove $\mathcal{A}^{\text {con }} \supseteq$ $\mathcal{A}^{\text {dis }},(8.8)$, and the theorem.

By Theorem 8.2, or section 3, any global attractor $\mathcal{A}_{\tilde{f}}$ in $\mathcal{A}^{\text {con }}$ can be realized as some attractor $\mathcal{A}_{f}$ in $\mathcal{A}^{\text {dis }}$ - for example by discretization. This raises the following open question: What is the minimal dimension $n+1$, for given $\mathcal{A}_{\tilde{f}}$, such that

$$
\mathcal{A}_{f} \cong \mathcal{A}_{\tilde{f}}
$$

for some dissipative Jacobi system $f=\left(f_{0}, f_{1}, \ldots, f_{n}\right)$ of form (8.7)? We recall from (3.9) that the canonical projection

$$
P: \quad \mathcal{A}_{\tilde{f}} \rightarrow \mathbb{R}^{n+1},
$$

$(P u)_{i}:=u\left(x_{i}\right), 0 \leq x_{1}<\ldots<x_{n} \leq 1$, is injective and differentiable, for

$$
n+1=\operatorname{dim} A_{\tilde{f}} \text {. }
$$

However, the flow induced by $P$ on $P \mathcal{A}_{\tilde{f}}$ need not be a tri-diagonal Jacobi system. Still the optimal bound (8.15) might be the answer to our question. In view of 
our proof of Theorem 8.2, the question reduces in principle to an investigation of realization of shooting permutations $\pi_{\tilde{f}}$ by discrete cumulative shootings.

In [FR96], we have introduced the weaker notion of connection equivalence. Here global attractors $\mathcal{A}_{f}, \mathcal{A}_{g}$ are called connection equivalent, $\mathcal{A}_{f} \sim \mathcal{A}_{g}$, if there exists a bijection $h: \mathcal{E}_{f} \rightarrow \mathcal{E}_{g}$ between the equilibria which preserves Morse index and the existence of heteroclinic connections. More precisely, there exists a heteroclinic connection from $u$ to $\tilde{u}$ in $\mathcal{A}_{f}$ if, and only if, there exists a connection from $h(u)$ to $h(\tilde{u})$ in $\mathcal{A}_{g}$. By definition,

$$
\mathcal{A}_{f} \cong \mathcal{A}_{g} \quad \Rightarrow \quad \mathcal{A}_{f} \sim \mathcal{A}_{g} .
$$

In [FR96], Corollary 6.1 we have noted that $\pi_{f}=\pi_{g}$ implies $\mathcal{A}_{f} \sim \mathcal{A}_{g}$. In [FR96], (6.11) we have conjectured that $\pi_{f}=\pi_{g}$ implies $\mathcal{A}_{f} \cong \mathcal{A}_{g}$; this is now proved in Theorems 1.1 and 8.1. We ask, this time, whether the converse of (8.16) also holds:

$$
\mathcal{A}_{f} \sim \mathcal{A}_{g} \quad \Rightarrow \quad \mathcal{A}_{f} \cong \mathcal{A}_{g} .
$$

This is nontrivial because, in [Fie94], [FR96], we have found shooting permutations $\pi_{f}, \pi_{g}$ with surprising connection equivalence $\mathcal{A}_{f} \sim \mathcal{A}_{g}$, in spite of $\pi_{f}, \pi_{g}$ not being even conjugate. The simplest examples require $N=9$ equilibria.

Although we have now examined orbit equivalence of global attractors in much detail, we are still lacking a good geometric description. Given $\pi_{f}$, we would like to be able to draw a "picture" of $\mathcal{A}_{f}$, on a reasonably descriptive level of abstraction. One approach would attempt to obtain a simplicial complex from the decomposition of $\mathcal{A}_{f}$ into equilibria and their manifolds of (transverse) heteroclinic connections. The combinatorics of such complexes, tied in with the shooting permutations $\pi_{f}$, might also hold the key to the orbit equivalence question (8.17). At the same time, it might shed some light on the surprising connection equivalences mentioned above. Providing good geometric attractor models which tie in with their dynamics remains a challenge!

\section{REFERENCES}

[AF88] S. Angenent and B. Fiedler. The dynamics of rotating waves in scalar reaction diffusion equations. Trans. Amer. Math. Soc., 307:545-568, (1988). MR 89h:35158

[Ama85] H. Amann. Global existence for semilinear parabolic systems. J. Reine Angew. Math., 360:47-83, (1985). MR 87b:35089

[Ang86] S. Angenent. The Morse-Smale property for a semi-linear parabolic equation. J. Diff. Eq., 62:427-442, (1986). MR 87e:58115

[Ang88] S. Angenent. The zero set of a solution of a parabolic equation. J. Reine Angew. Math., 390:79-96, (1988). MR 89j:35015

[Ang90] S. Angenent. Parabolic equations for curves on surfaces. I. curves with $p$-integrable curvature. Ann. Math., 132:451-483, (1990). MR 91k:35102

[Ang91] S. Angenent. Parabolic equations for curves on surfaces. II. intersections, blow-up and generalized solutions. Ann. Math., 133:171-215, (1991). MR 92b:58039

[Ari75] R. Aris. The Mathematical Theory of Diffusion and Reaction in Permeable Catalysts I, II. Clarendon Press, Oxford, 1975.

[BF88] P. Brunovský and B. Fiedler. Connecting orbits in scalar reaction diffusion equations. Dynamics Reported, 1:57-89, (1988). MR 89f:58071

[BF89] P. Brunovský and B. Fiedler. Connecting orbits in scalar reaction diffusion equations II: The complete solution. J. Diff. Eq., 81:106-135, (1989). MR 91d:35111

[BP87] P. Brunovský and P. Poláčik. Generic hyperbolicity for reaction diffusion equations on symmetric domains. J. Appl. Math. Phys., 38:172-183, (1987). MR 88d:35102

[BV89] A.V. Babin and M.I. Vishik. Attractors in Evolutionary Equations. Nauka, Moscow, 1989. MR 92f:58101 
[Cer68] J. Cerf. Sur les difféomorphismes de la sphère de dimension trois $\left(\Gamma_{4}=0\right)$. Springer Verlag, Berlin, 1968. Lect. Notes in Math., vol. 53. MR 37:4824

[Cha74] N. Chafee. A stability analysis for a semilinear parabolic partial differential equation. J. Diff. Eq., 15:522-540, (1974). MR 50:10507

[CI74] N. Chafee and E. Infante. A bifurcation problem for a nonlinear parabolic equation. J. Applicable Analysis, 4:17-37, (1974). MR 55:13084

[CP89] J. Carr and R.L. Pego. Metastable patterns in solutions of $u_{t}=\epsilon^{2} u_{x x}-f(u)$. Comm. Pure Appl. Math., 42, 523-576, (1989). MR 90f:35091

[CS80] C. C. Conley and J. Smoller. Topological techniques in reaction diffusion equations. Springer Lect. Notes in Biomath., 38:473-483, (1980). MR 82j:35014

[Fen79] N. Fenichel. Geometric singular perturbation theory for ordinary differential equations. J. Diff. Eq., 31:53-98, (1979). MR 80m:58032

[FH89] G. Fusco and J.K. Hale. Slow-motion manifolds, dormant instability, and singular perturbations. J. Dyn. Diff. Eq., 1:75-94, (1989). MR 90i:35131

[Fie89] B. Fiedler. Discrete Ljapunov functionals and $\omega$-limit sets. Math. Mod. Num. Anal., 23:415-431, (1989). MR 91g:58166

[Fie94] B. Fiedler. Global attractors of one-dimensional parabolic equations: sixteen examples. Tatra Mountains Math. Publ., 4:67-92, (1994). MR 95g:35089

[Fie96] B. Fiedler. Do global attractors depend on boundary conditions? Doc. Math. J. DMV, 1:215-228, (1996). MR 97g:58099

[FO88] G. Fusco and W.M. Oliva. Jacobi matrices and transversality. Proc. Royal Soc. Edinburgh A, 109:231-243, (1988). MR 89k:58229

[FR91] G. Fusco and C. Rocha. A permutation related to the dynamics of a scalar parabolic PDE. J. Diff. Eq., 91:111-137, (1991). MR 92h:35008

[FR96] B. Fiedler and C. Rocha. Heteroclinic orbits of semilinear parabolic equations. J. Diff. Eq., 125:239-281, (1996). MR 96k:58200

[FR97] B. Fiedler and C. Rocha. Boundary permutations and global attractors of dissipative Jacobi systems. In preparation, (1997).

[FR98] B. Fiedler and C. Rocha. Realization of meander permutations by boundary value problems. Preprint, (1998).

[Hal88] J.K. Hale. Asymptotic Behavior of Dissipative Systems. Math. Surv. 25. AMS Publications, Providence, 1988. MR 89g:58059

[Hal94] J.K. Hale. Numerical dynamics. Contemporary Math., 172:1-30, (1994). MR 95g:58147

[Hen81] D. Henry. Geometric Theory of Semilinear Parabolic Equations. Lect. Notes Math. 840. Springer Verlag, New York, 1981. MR 83j:35084

[Hen85] D. Henry. Some infinite dimensional Morse-Smale systems defined by parabolic differential equations. J. Diff. Eq., 59:165-205, (1985). MR 86m:58080

[Hir88] M. W. Hirsch. Stability and convergence in strongly monotone dynamical systems. J. Reine Angew. Math., 383:1-58, (1988). MR 89c:58108

[HMO84] J.K. Hale, L.T. Magalhães, and W.M. Oliva. An Introduction to Infinite Dimensional Dynamical Systems - Geometric Theory. Springer Verlag, New York, 1984. MR 86h:34001

[Lad91] O.A. Ladyzhenskaya. Attractors for Semi-Groups and Evolution Equations. Cambridge University Press, 1991. MR 92k:58040

[Mat82] H. Matano. Nonincrease of the lap-number of a solution for a one-dimensional semilinear parabolic equation. J. Fac. Sci. Univ. Tokyo Sec. IA, 29:401-441, (1982). MR 84m:35060

[Mat86] H. Matano. Strongly order-preserving local semi-dynamical systems - theory and applications. In F. Kappel H. Brezis, M.G. Crandall, editor, Semigroups, Theory and Applications, pages 178-185. John Wiley \& Sons, New York, 1986. MR 88d:54053

[Mat88] H. Matano. Asymptotic behavior of solutions of semilinear heat equations on $S^{1}$. In J. Serrin W.-M. Ni, L.A. Peletier, editor, Nonlinear Diffusion Equations and their Equilibrium States II, pages 139-162. Springer Verlag, New York, 1988. MR 90b:35033

[Mat95] H. Matano. Personal communication, (1995).

[MPS88] J. Mallet-Paret and G. Sell. Inertial manifolds for reaction diffusion equations in higher space dimensions. J. Amer. Math. Soc., 1:805-866, (1988). MR 90h:58056 
[MPS90] J. Mallet-Paret and H. Smith. The Poincaré-Bendixson theorem for monotone cyclic feedback systems. J. Diff. Eq., 4:367-421, (1990). MR 91k:58098

[Oli83] W.M. Oliva. Stability of Morse-Smale maps. Rel. Tec, IME-USP, São Paulo, MAP 0301, (1983).

[Pal69] J. Palis. On Morse-Smale dynamical systems. Topology, 8:385-404, (1969). MR 39:7620

[Paz83] A. Pazy. Semigroups of Linear Operators and Applications to Partial Differential Equations. Springer Verlag, New York, 1983. MR 85g:47061

[PdM82] J. Palis and W. de Melo. Geometric Theory of Dynamical Systems. Springer Verlag, New York, 1982. MR 84a:58004

[Pol33] G. Polya. Qualitatives über Wärmeaustausch. Z. Angew. Math. Mech., 13:125-128, (1933).

[PS70] J. Palis and S. Smale. Structural stability theorems. In Global Analysis. Proc. Symp. in Pure Math. vol. XIV. AMS, Providence, 1970. MR 42:2505

[Rau95] G. Raugel. Personal communication, (1995).

[Roc91] C. Rocha. Properties of the attractor of a scalar parabolic PDE. J. Dyn. Diff. Eq., 3:575-591, (1991). MR 92m:35122

[Roc94] C. Rocha. Bifurcations in discretized reaction-diffusion equations. Resenhas IME-USP, 1:403-419, (1994). MR 96h:58160

[SGKM95] A.A. Samarskii, V.A. Galaktionov, S.P. Kurdyumov, and A.P. Mikhailov. Blow-Up in Quasilinear Parabolic Equations. Walter de Gruyter \& Co., Berlin, 1995. MR 96b:35003

[Smo83] J. Smoller. Shock Waves and Reaction-Diffusion Equations. Springer Verlag, New York, 1983. MR 84d:35002

[Stu36] C. Sturm. Sur une classe d'équations à différences partielles. J. Math. Pure Appl., 1:373-444, (1836).

[Tem88] R. Temam. Infinite Dimensional Dynamical Systems in Mechanics and Physics. Springer Verlag, New York, 1988. MR 89m:58056

[Wol92] G. Wolansky. Stationary and quasi-stationary shock waves for non-spatially homogeneous Burger's equation in the limit of small dissipation. I. Indiana Univ. Math. J., 41:43-69, (1992). MR 93h:35185

[Wol94] G. Wolansky. On the slow evolution of quasi-stationary shock waves, J. Dyn. Differ. Equations, 6:247-276, (1994). MR 95c:35035

[Zel68] T.I. Zelenyak. Stabilization of solutions of boundary value problems for a second order parabolic equation with one space variable. Differential Equations, 4:17-22, (1968). MR 36:6806

Institut für Mathematik I, Freie Universität Berlin, Arnimallee 2-6, D-14195 Berlin, GERMANY

Departamento de Matemática, Instituto Superior técnico, Avenida Rovisco Pais, 1096 Lisboa Codex, Portugal 